\title{
Knowledge Gaps and Recommendations for Future Research of Indoor Particulate Matter in Poland
}

\author{
Karolina Kuskowska ${ }^{1 *}$, Wioletta Rogula-Kozłowska², \\ Kamila Widziewicz ${ }^{3}$, Patrycja Rogula-Kopiec ${ }^{3}$ \\ ${ }^{1}$ Faculty of Civil Safety Engineering, The Main School of Fire Service, Warsaw, Poland \\ ${ }^{2}$ Faculty of Fire Safety Engineering, The Main School of Fire Service, Warsaw, Poland \\ ${ }^{3}$ Institute of Environmental Engineering, Polish Academy of Sciences, Zabrze, Poland
}

Received: 18 April 2018

Accepted: 23 July 2018

\begin{abstract}
Research concerning the ambient particulate matter (PM) in the indoor environment has attracted much interest lately. Most often, studies concern some aspect of PM mass concentrations for the $\mathrm{PM}_{10}$ and $\mathrm{PM}_{2.5}$ fractions and less often the chemical composition of the indoor PM. In the framework of this study, an overview of the existing data in the literature concerning PM in the indoor environment of non-residential buildings has been compiled. An in-depth literature review indicates a lack of comprehensive research data regarding the state and quality of atmospheric air in non-residential buildings. It also highlights an emerging need for more knowledge on the indoor/outdoor air pollution relationships in such facilities. Although several studies underline the topics connected to the concentrations and chemical properties of PM in public utility environments, like offices, kindergartens, schools, churches, libraries, or in occupational environments, only a limited number of those are concerned with its presence inside sports facilities. The concentration of PM in the indoor air of closed sports venues is an important parameter for the users of these facilities due to the potentially harmful effects associated with PM inhalation. This negative influence includes the loss of athletic performance and health reflected by, among other factors, the loss of lung capacity and decreased lung function.
\end{abstract}

Keywords: $\mathrm{PM}_{10} ; \mathrm{PM}_{2.5}$; indoor air quality; indoor/outdoor; sport facilities

\section{Introduction}

Ambient particulate matter (PM) is a key air pollutant due to both the existence of epidemiological evidence which links exposure to PM with various

*e-mail: kkuskowska@sgsp.edu.pl health effects and the fact that so far, in many urbanized areas of the world, the concentrations of PM still cannot be sufficiently reduced despite the existence of air quality standards and many public policies, programmes, and guidelines. Much epidemiological data show that short-term as well as long-term exposure to high concentrations of PM increases the risk of cardiovascular, respiratory, and nervous system diseases. Generally, each $10 \mu \mathrm{g} / \mathrm{m}^{3}$ increase in the daily concentrations 
of $\mathrm{PM}_{10}\left(\mathrm{PM}_{\mathrm{x}}-\right.$ particles with aerodynamic diameter $\leq \mathrm{x} \mu \mathrm{m})$ may be associated with an increased risk of certain health effects [1]. As research has not identified the PM thresholds below which adverse effects do not occur it is difficult to determine what levels of PM pollution are in fact dangerous for humans [2-3]. However, certain concentrations exceeding the daily limits established individually in each country (or group of countries) should be considered as high.

In general, the negative influence of PM on human health can be observed by the occurrence of the following effects: aggravation of asthma, increased coughing, breathing problems, chronic bronchitis, lung function decline, and premature death. When particles are inhaled or swallowed they may cause a decrease in lung function, coughing, shortness of breath, asthma attacks, chronic lung disease, cardiovascular disease, or even the development of cancer (if particles contain carcinogenic substances) [4-6]. It should, however, be remembered that these symptoms do not occur under the sole influence of PM. Usually, PM affects the human body along with other factors, the effects of which may deepen or aggravate the effects of PM. Epidemiological studies demonstrate the relationships between an increase in PM concentrations in the atmosphere and increased frequency of daily emergency room visits due to asthma and lower respiratory symptoms. The most frequent symptoms were observed in patients with chronic heart disease, lung disease, and pneumonia, which suggests that the occurrence of these disorders could be a co-founding risk factor under high PM concentrations [7]. The relationships between suspended particles and health effects, including mortality, are determined at ever lower concentration levels for PM [810]. Although it is not clear whether the PM size or the number concentration [11], chemical [12] or biological composition, or physical properties (mass loading, number of particles, total area, or electrostatic properties) [2] is the more decisive factor [13], the negative health effects of PM always appear in the exposed population. The strongest relationships between air pollution and health outcomes in the population are observed in the case of $\mathrm{PM}_{10}$ and $\mathrm{PM}_{2.5}$ [2]. Health effects caused by fine particles with aerodynamic diameters between 0.1 and $2.5 \mu \mathrm{m}$ and ultrafine ones with diameters between 0.01 and $0.1 \mu \mathrm{m}$ are related to their number concentrations more so than to the PM mass [14].

Research on the presence of PM in the atmosphere is conducted by various institutions and scientific research units all around the world. In Poland, the last decade has resulted in the creation of several dozen important scientific works concerning PM characteristics. On the basis of these works, it is possible to assess not only the degree of air pollution by PM in various regions of Poland, but also:

- The characterization of PM fractions in various areas together with a statistical assessment of PM mass distribution and number distribution in relation to PM size [15-17].
- The chemical composition of different PM fractions [18], together with the assessment of the presence of toxic and carcinogenic compounds in selected PM fractions [18-21].

- The origin of PM in different regions and in different seasons, taking into account the impact of specific or typical emission sources, thermodynamic conditions of the atmosphere, or other parameters such as the impact of the long-distance transport of air pollutants [22-31].

- The impact of PM and its components on human health [32-39].

Due to the fact that people spend a lot of time inside buildings such as flats, offices, schools, shopping centres, etc., more and more attention is currently being paid to PM characteristics in the indoor environment and the different factors modulating its concentration and composition in various types of rooms. In general, the most ground-breaking works regarding PM in living quarters were conducted in the 1990s [4046]. And although to this day research on air quality inside building interiors, apartments, houses, etc., are conducted all over the world, some of the most important facts about PM in the indoor environment have already been established, and newly conducted studies are rather a complement to the existing base of knowledge about PM than a source of new information of general importance. In general, it has been established beyond any doubt that PM concentrations, especially those of fine PM in homes and houses, are usually higher or almost equal to those usually met in atmospheric air. Two factors are generally responsible for such a distribution of PM concentration: 1) free migration of outdoor PM inside the rooms and 2) the presence of various internal sources of PM emissions, i.e., household furnaces, chemicals, carpets, animals, etc. Therefore, it is obvious that beyond such determinants of the quantity and quality of indoor PM like internal emission sources, atmospheric and meteorological parameters are no less important.

Due to the various types of services provided inside public utilities and therefore the diversity of internal sources of PM and its gaseous precursors, both the chemical and physical properties of PM inside such facilities might be more complex than in the case of atmospheric PM. With the exception of facilities equipped with the simplest mechanical ventilation or high-efficiency air conditioning and purification systems, the importance of outdoor PM on the quality of indoor air is of great importance and should be quantified each time.

In terms of the PM pollution levels, Poland differs from other European countries. In fact, the levels of PM pollution (especially the levels of fine PM) registered in Polish cities exceed by even several times the concentrations usually met in other urbanized areas of Europe. Paradoxically, research concerning the presence of PM in the indoor air of living quarters in Poland is rather rare. In principle, there is no database 
and no standard concerning PM in living quarters in Poland. However, there is relatively rich data on air pollution in various non-residential premises like public utilities [47-53], some of which is information derived from measurements of the relationships between indoor and outdoor PM. This article contains a comprehensive review of different studies on PM in the indoor air of non-residential and non-industrial facilities with a particular emphasis on the Polish scientific literature. This study draws attention to a special type of room - closed sports facilities - in which comprehensive research on PM pollution has not been conducted so far.

\section{Results and Discussion}

The number of publications on indoor air quality, as in the case of outdoor air, is very high (Fig. 1). Over the last decade, the intensity of publishing research devoted to PM in closed rooms has increased by at least several times. The situation is different in Poland, where the results of comprehensive PM research in various types of closed facilities have emerged into the international arena only within the last few years. Both the abovementioned facts have an impact on 1) the ever-growing number of articles on PM in indoor air all around the world and 2) widely disseminated information

Table 1. PM levels in closed non-residential facilities - selected world publications.

\begin{tabular}{|c|c|c|c|c|c|}
\hline No. & Ref. & $\begin{array}{l}\text { Country, sampling } \\
\text { site }\end{array}$ & $\begin{array}{l}\text { Measurement } \\
\text { period }\end{array}$ & $\begin{array}{l}\text { PM fraction and analy- } \\
\text { sis method }\end{array}$ & Results/comments \\
\hline 1. & {$[54]$} & $\begin{array}{l}\text { Portugal, a total of } \\
\text { nine classrooms } \\
\text { were monitored, } \\
\text { eight integrated in } \\
\text { elementary schools } \\
\text { and one in a kin- } \\
\text { dergarten }\end{array}$ & $\begin{array}{l}\text { 9-29 Janu- } \\
\text { ary 2012; 6-h } \\
\text { measurements }\end{array}$ & $\begin{array}{c}\mathrm{PM}_{10} \text { concentration } \\
\text { by light scattering } \\
\text { technique; } \mathrm{PM}_{2.5} \\
\text { concentration by the } \\
\text { gravimetric method. } \\
\text { Additional measure- } \\
\text { ments of temperature, } \\
\text { relative humidity } \\
\text { (RH), } \mathrm{CO}_{2}, \mathrm{CO} \text {, and } \\
\text { total volatile organic } \\
\text { compounds (TVOCs). }\end{array}$ & $\begin{array}{l}\text { The research has shown that the source of high con- } \\
\text { centrations of PM was, to a large extent, due to a faulty } \\
\text { ventilation system. Continuous measurements of } \mathrm{PM}_{10} \\
\text { suggest that the physical activity of pupils (greater for } \\
\text { older children than in case of younger children), contrib- } \\
\text { utes to the re-suspension of dust particles and therefore } \\
\text { their increased deposition on underlying surfaces. }\end{array}$ \\
\hline 2. & {$[55]$} & $\begin{array}{l}\text { Homes, offices and } \\
\text { surroundings in } \\
\text { Canadian cities }\end{array}$ & $1999-2010$ & $\begin{array}{l}\mathrm{PM}_{10} \text { and } \mathrm{PM}_{2.5} \text { by } \\
\text { optical and gravimetric } \\
\text { methods. }\end{array}$ & $\begin{array}{l}\text { The research was conducted as part of a national research } \\
\text { project on air quality and was carried out in selected } \\
\text { offices and homes. At the end of the project, a guide was } \\
\text { created on how to reduce the high concentration of PM } \\
\text { suspended in indoor air. }\end{array}$ \\
\hline 3. & {$[56]$} & $\begin{array}{l}\text { USA, office build- } \\
\text { ings and their } \\
\text { surroundings }\end{array}$ & $\begin{array}{c}\text { 2012, 24-h } \\
\text { measurements }\end{array}$ & $\begin{array}{c}\text { Low-cost optical } \\
\text { PM sensors (Total } \\
\text { Suspended Particles - } \\
\text { TSP). }\end{array}$ & $\begin{array}{l}\text { The experiment was carried out in the main corridor of } \\
\text { Cory Hall on University of California Berkeley campus. } \\
\text { The results showed that local human activity, measured } \\
\text { visually by a camera, was correlated with the concentra- } \\
\text { tion of coarse particles, particularly those } \geq 2.5 \mu \mathrm{m} \text {. }\end{array}$ \\
\hline 4. & {$[57]$} & $\begin{array}{l}\text { Iran, primary } \\
\text { schools and their } \\
\text { surroundings }\end{array}$ & $\begin{array}{l}\text { October } 2011- \\
\text { June } 2012 ; 26- \\
\text { day campaign, } \\
5.5 \text {-h measure- } \\
\quad \text { ments }\end{array}$ & $\begin{array}{c}\mathrm{PM}_{1}, \mathrm{PM}_{2.5} \text {, and } \mathrm{PM}_{10} \\
\text { by optical methods } \\
\text { with parallel gravimet- } \\
\text { ric methods. }\end{array}$ & $\begin{array}{l}\text { In schools located near both main and small roads, the } \\
\text { association between indoor fine particle }\left(\mathrm{PM}_{2.5} \text { and } \mathrm{PM}_{1}\right) \\
\text { and outdoor } \mathrm{PM}_{2.5} \text { levels was stronger than that between } \\
\text { indoor } \mathrm{PM}_{10} \text { and outdoor } \mathrm{PM}_{2.5} \text { levels. }\end{array}$ \\
\hline 5. & {$[58]$} & $\begin{array}{l}\text { Finland, kitchen in } \\
\text { an apartment and } \\
\text { lecture room inside } \\
\text { the university }\end{array}$ & $\begin{array}{l}\text { September } \\
2013\end{array}$ & $\begin{array}{c}\mathrm{PM}_{2.5}, \mathrm{PM}_{10} \text { by opti- } \\
\text { cal and gravimetric } \\
\text { methods. }\end{array}$ & $\begin{array}{l}\text { The article compares the concentration of PM inside } \\
\text { (I) selected types of rooms and outside (O) and also } \\
\text { compares the concentration of pollutants depending on } \\
\text { the ventilation solution (no ventilation in the kitchen, me- } \\
\text { chanical ventilation in classroom). The results obtained } \\
\text { indicate that the lowest I/O ratio according to the results } \\
\text { in the apartment was with the window open and the fan } \\
\text { switched on, whereas the best result in the classroom } \\
\text { was with the mechanical ventilation system with filter } \\
\text { switched on and the window open. }\end{array}$ \\
\hline 6. & [59] & $\begin{array}{c}\text { Brasil, construction } \\
\text { jobsites }\end{array}$ & $\begin{array}{l}\text { 10-day meas- } \\
\text { urements dur- } \\
\text { ing construc- } \\
\text { tion activities } \\
\text { (earthworks, } \\
\text { superstructure } \\
\text { and finishing) }\end{array}$ & $\begin{array}{l}\mathrm{PM}_{2.5}, \mathrm{PM}_{10} \text {, and } \\
\text { TSP by gravimetric } \\
\text { methods. }\end{array}$ & $\begin{array}{l}\text { The findings show that the activities on construction } \\
\text { sites emit different types of particles with environmental } \\
\text { impacts through a higher emission of TSP. The concentra- } \\
\text { tion values obtained for PM } \mathrm{P}_{10} \text { in this study did not exceed } \\
\text { the limits set by the Brazilian standards; however, some } \\
\text { of the concentration levels measured exceeded the stand- } \\
\text { ards set by the World Health Organization (WHO). }\end{array}$ \\
\hline
\end{tabular}


Table 1. Cotinued.

\begin{tabular}{|c|c|c|c|c|c|}
\hline 7. & [60] & $\begin{array}{l}\text { United Arab Emir- } \\
\text { ates (UAE), } 628 \\
\text { residences }\end{array}$ & $\begin{array}{l}\text { 5-month long } \\
\text { campaign, } \\
\text { each measure- } \\
\text { ment lasting } 7 \\
\text { days }\end{array}$ & $\begin{array}{c}\text { TSP, } \mathrm{PM}_{10}, \mathrm{PM}_{2.5} \text {, } \\
\text { and ultrafine PMby } \\
\text { UNC Passive Aerosol } \\
\text { Sampler. }\end{array}$ & $\begin{array}{l}\text { Overall, indoor air quality in UAE residences is quite } \\
\text { similar to other developed nations; concentrations of } \\
\text { particles were lower indoors than outdoors. Indoor PM } \\
\text { concentrations were higher in rural areas than urban loca- } \\
\text { tions, but outdoor PM concentrations were not signifi- } \\
\text { cantly different between rural and urban locations. Indoor } \\
\text { concentrations of PM } \text { PM }_{2.5} \text { and } \text { PM }_{10} \text { were significantly } \\
\text { associated with vehicles being parked within five metres } \\
\text { of the home, with the use of central air conditioning, and } \\
\text { with kitchens that were located within the residence. }\end{array}$ \\
\hline 8. & [61] & $\begin{array}{l}\text { Malaysia, office } \\
\text { buildings and sur- } \\
\text { roundings }\end{array}$ & $\begin{array}{l}\text { 8-h measure- } \\
\text { ments during } \\
\text { working days }\end{array}$ & $\begin{array}{l}\mathrm{PM}_{2.5}, \mathrm{PM}_{10} \text { by the } \\
\text { gravimetric method. }\end{array}$ & $\begin{array}{l}\text { The results presented indicate high concentrations of } \\
\text { respirable and inhalable PM inside offices. Such a situ- } \\
\text { ation could contribute to increased exposure to solid } \\
\text { particles suspended in indoor air. This could lead to nega- } \\
\text { tive health effects among office occupants which should } \\
\text { stimulate building services to overcome these problems } \\
\text { and take action to develop awareness among staff about } \\
\text { health and safety in office buildings, especially regarding } \\
\text { airborne particulates. }\end{array}$ \\
\hline 9. & [62] & $\begin{array}{l}\text { Malaysia, univer- } \\
\text { sity building }\end{array}$ & $\begin{array}{l}\text { Measurement } \\
\text { period June- } \\
\text { December } \\
\text { 2012; between } \\
\text { 9:00 and } \\
\text { 17:30 each } \\
\text { day }\end{array}$ & $\begin{array}{l}\mathrm{PM}_{10} \text { by the gravimet- } \\
\text { ric method. }\end{array}$ & $\begin{array}{l}\text { The results presented indicate a high contamination } \\
\text { of indoor air pollution in the Biology department of } \\
\text { Malaysian University. It was found that the presence of } \\
\text { indoor-related PM originated from the poorly main- } \\
\text { tained ventilation system, the activity of occupants, and } \\
\text { typical office equipment such as printers and photocopy } \\
\text { machines. }\end{array}$ \\
\hline
\end{tabular}

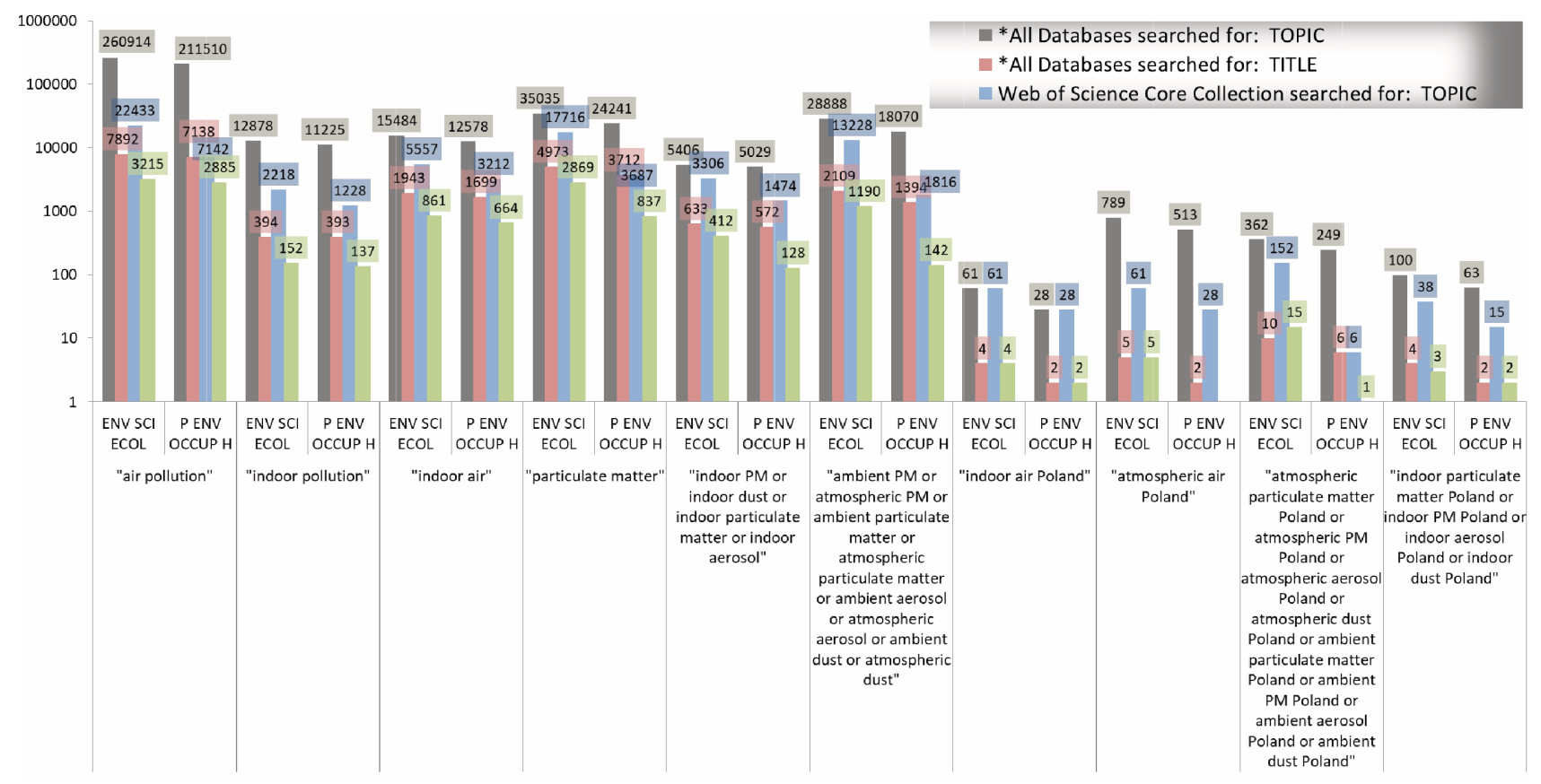

Fig. 1. Number (Y axis) of studies (including articles, reviews, corrections, books, proceedings papers, editorial materials, letters, reprints, meeting abstracts, book chapters, news item, retracted publication) published in peer-reviewed journals and indexed in All Databases (which includes the Science Citation Index: Web of Science Core Collection BIOSIS Citation Index Current Contents Connect, Data Citation Index, Derwent Innovations Index, KCI-Korean Journal Database, MEDLINE, Russian Science Citation Index, SciELO Citation Index, Zoological Record) and in Web of Science Core Collection only, assigned to the following subject categories: Environmental Science (Web of Science Core Collection) or Environmental Science Ecology (All Databases) and Public, Environmental and Occupational Health (X axis: ENV SCI ECOL; P ENV OCCUP H). Studies are identified through periodic searches using the specific search criteria (X axis). For this review, studies indexed from January 2007 up to June 2018 were included. 


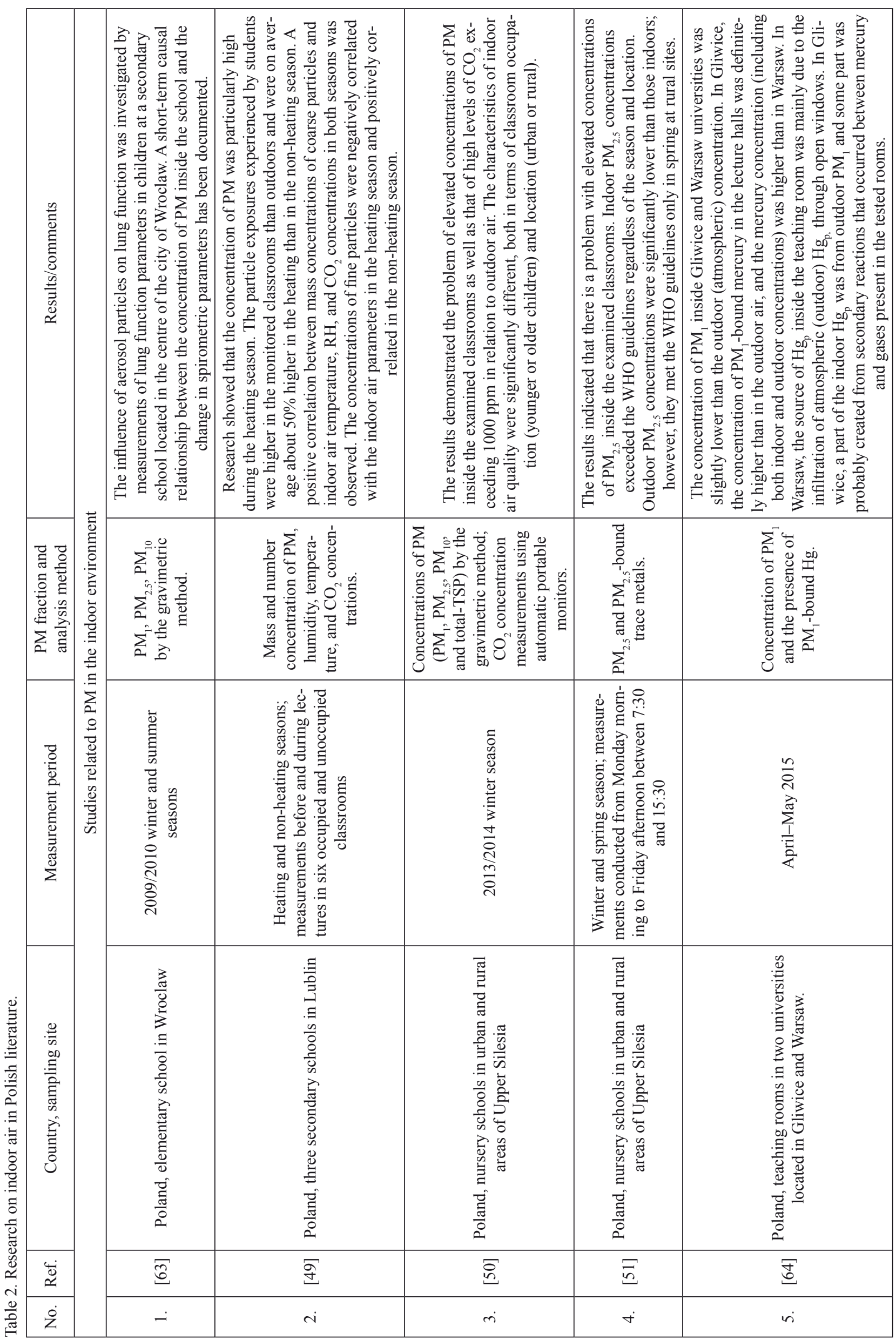




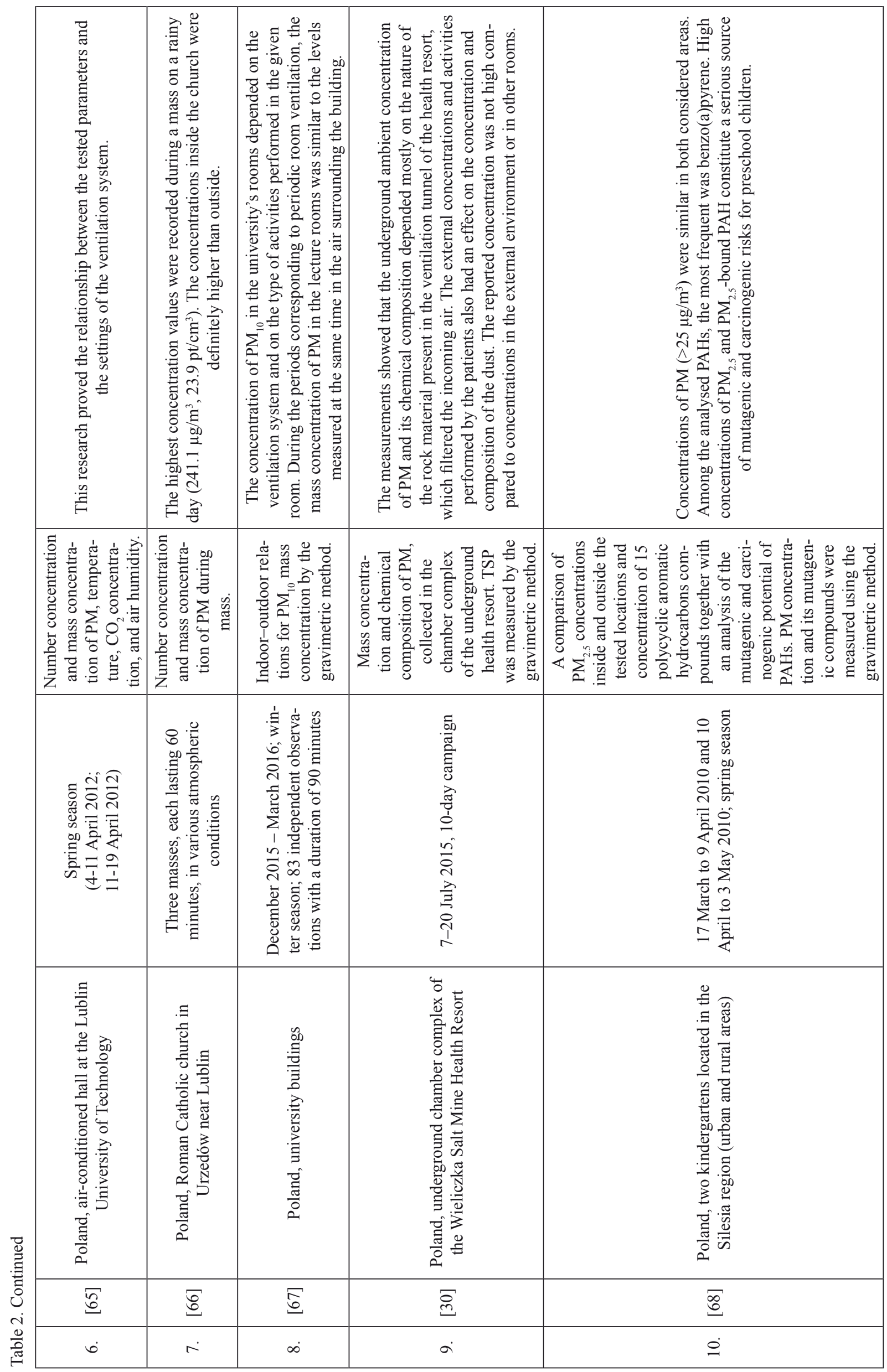




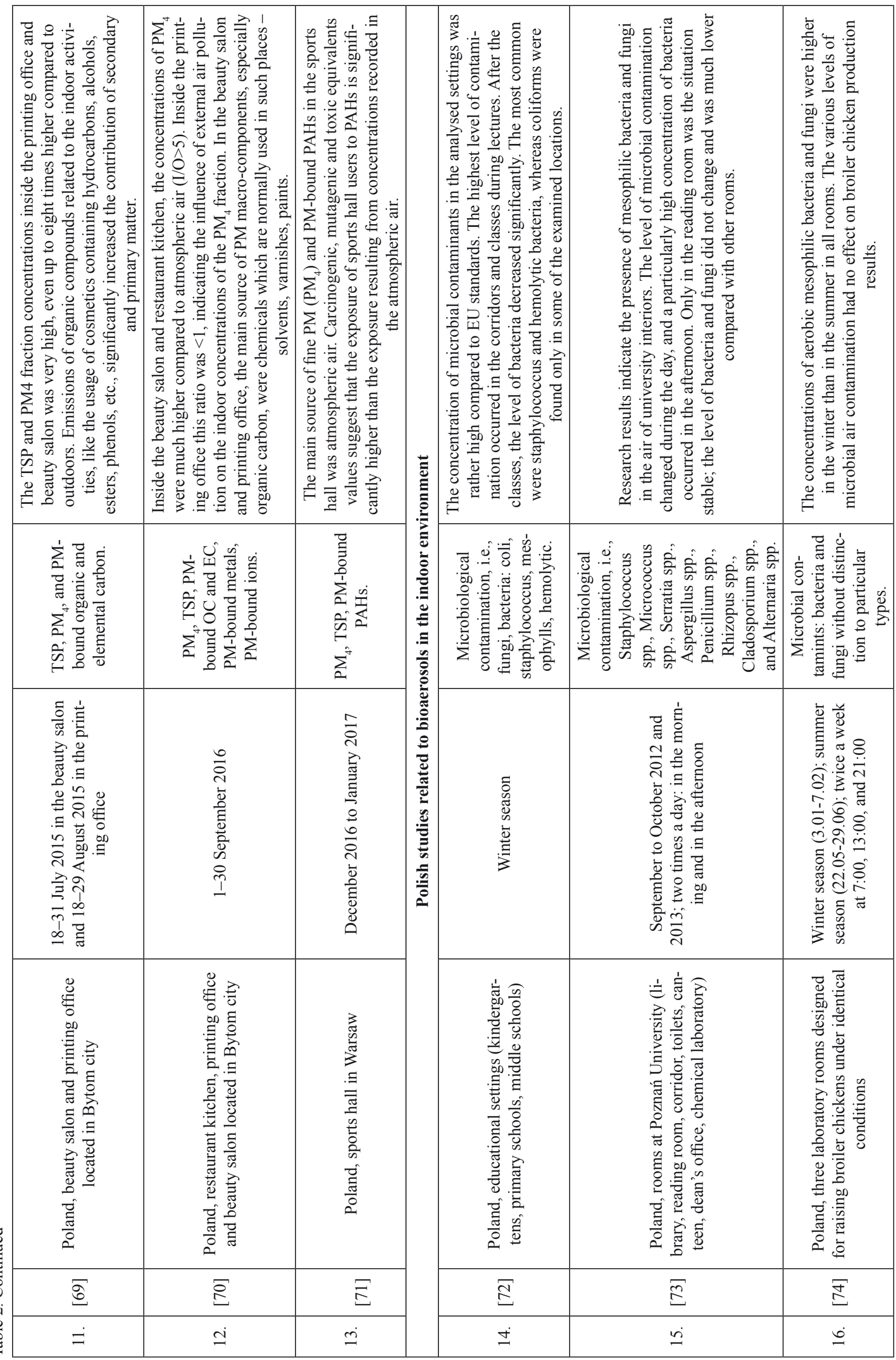




\begin{tabular}{|c|c|c|c|c|c|c|}
\hline 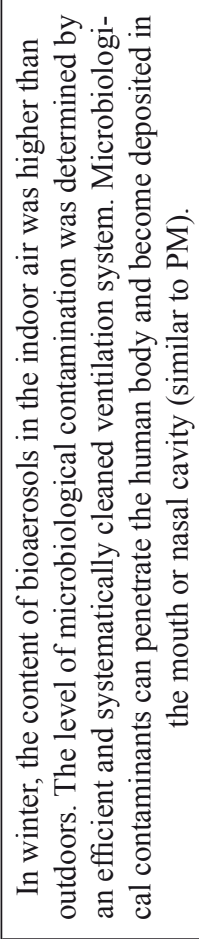 & 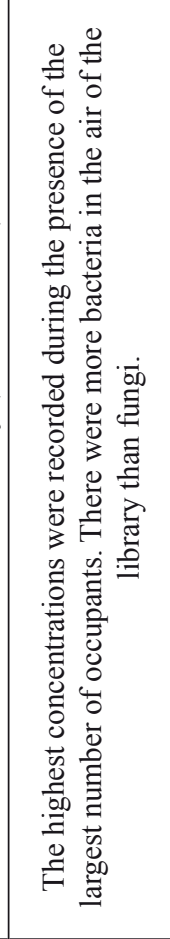 & 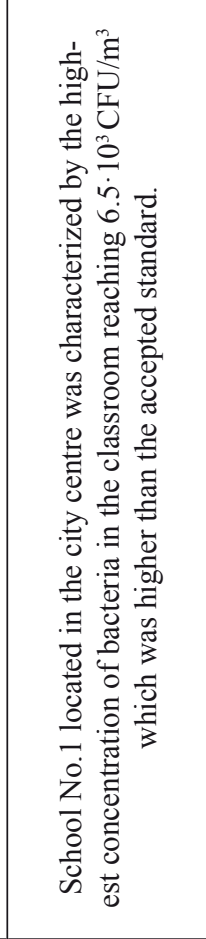 & 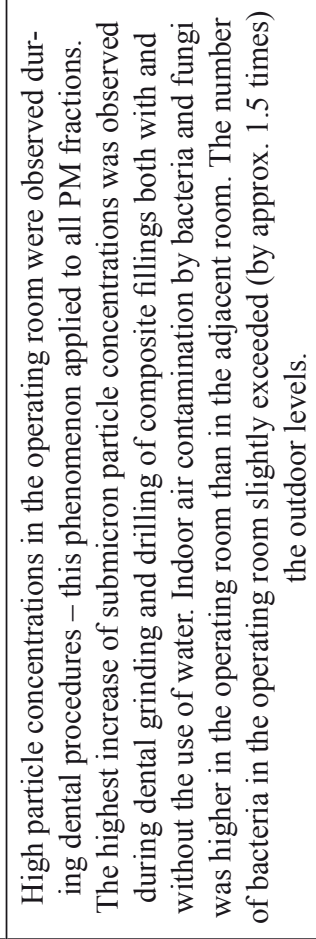 & 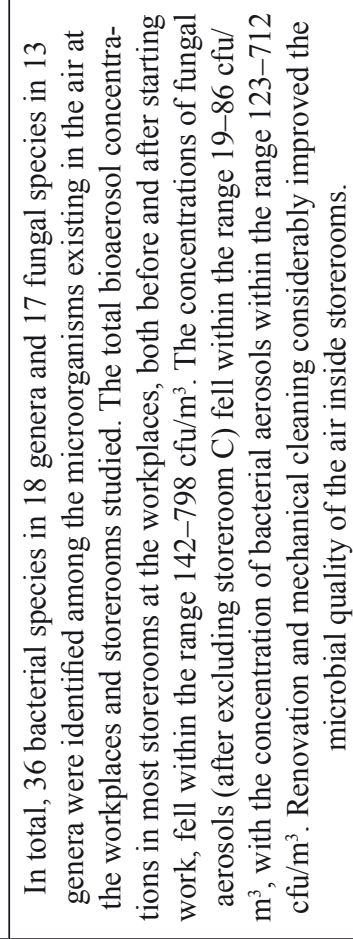 & 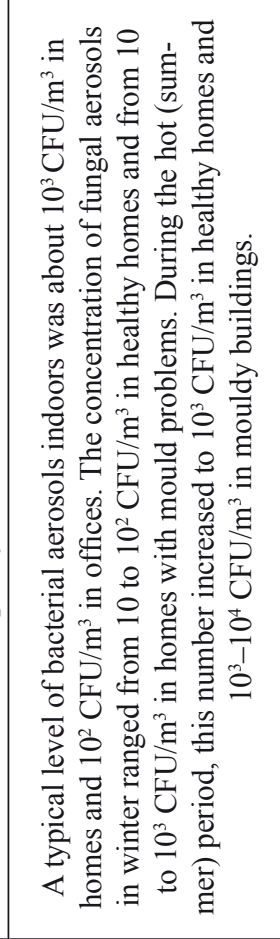 & 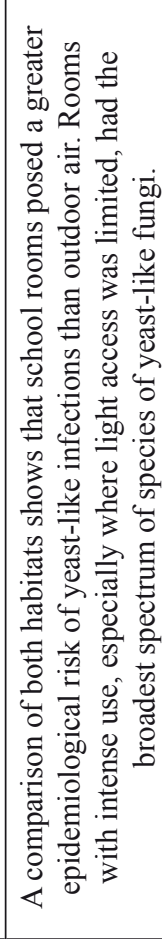 \\
\hline 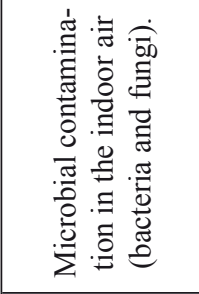 & 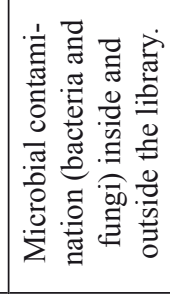 & 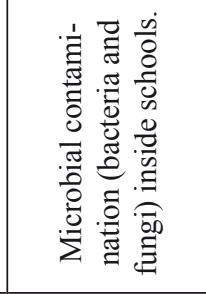 & 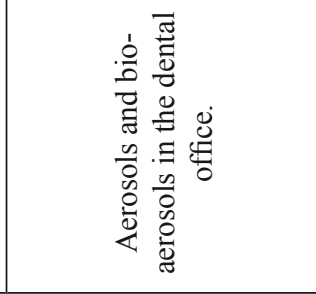 & 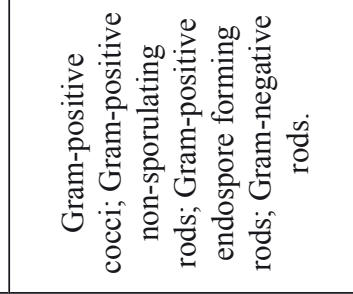 & 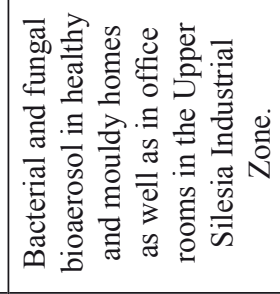 & 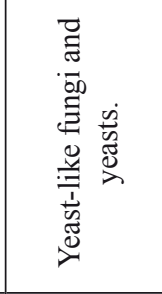 \\
\hline 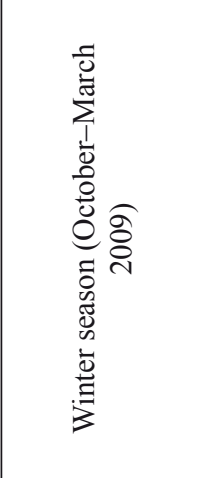 & 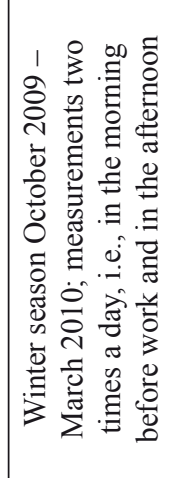 & 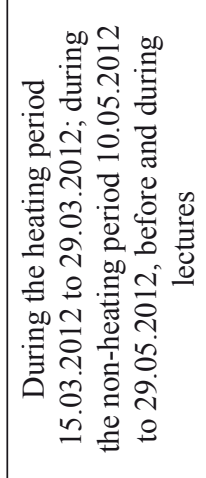 & 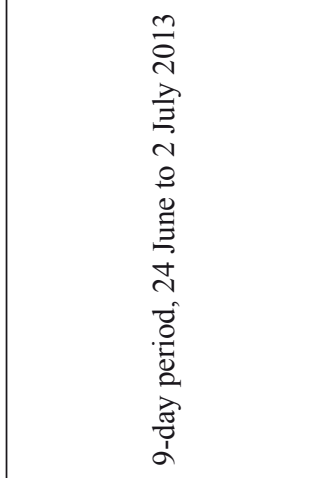 & 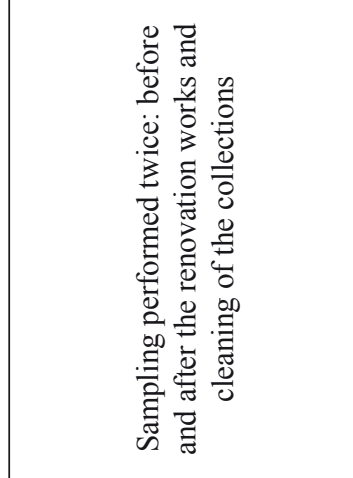 & 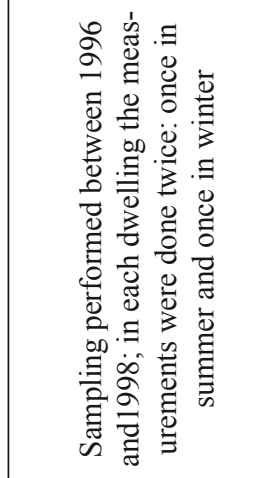 & 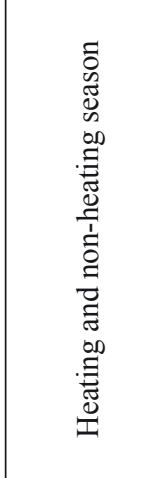 \\
\hline 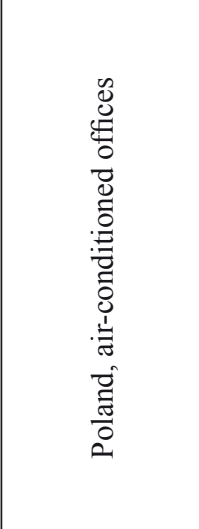 & 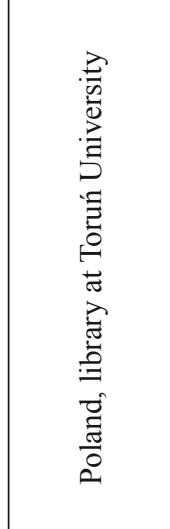 & 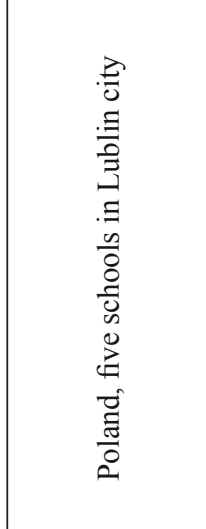 & 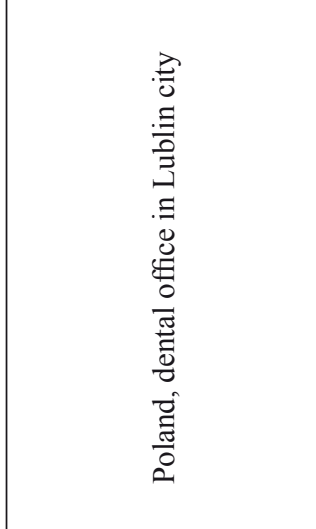 & 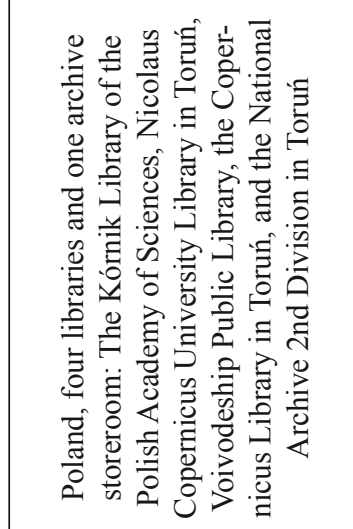 & 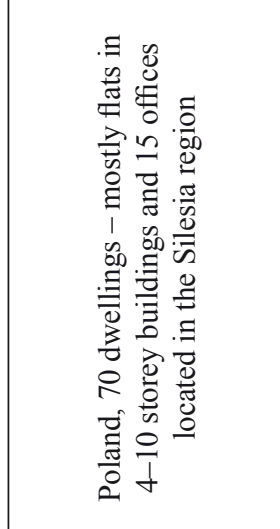 & 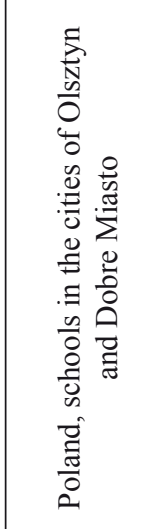 \\
\hline$\sqrt[n]{ }$ & $\stackrel{\Xi}{\Xi}$ & $\mathbb{E}$ & $\stackrel{\infty}{=}$ & $\bar{\Xi}$ & $\bar{\infty}$ & $\overline{\bar{\infty}}$ \\
\hline$\therefore$ & $\dot{\infty}$ & $\stackrel{\circ}{\circ}$ & $\dot{i}$ & $\dot{\vec{N}}$ & તี & $\dot{\lambda}$ \\
\hline
\end{tabular}




\begin{tabular}{|c|c|c|c|c|c|}
\hline 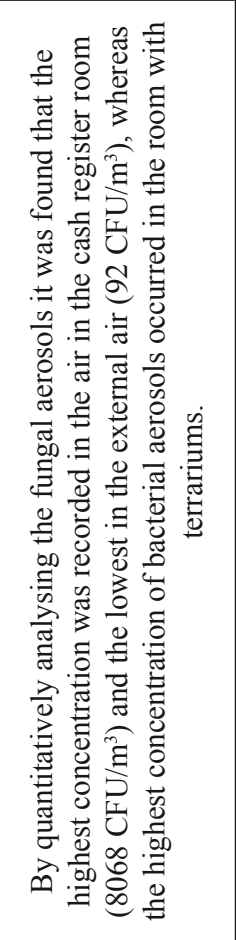 & 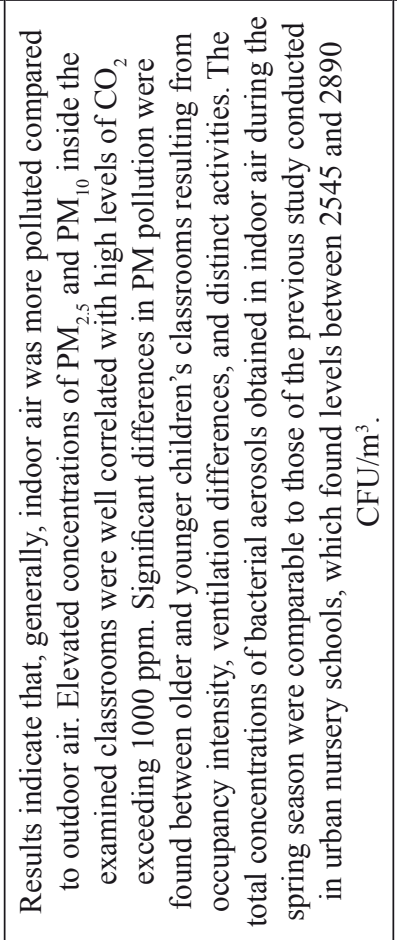 & 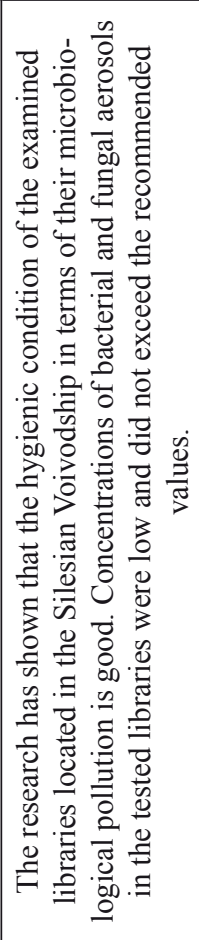 & 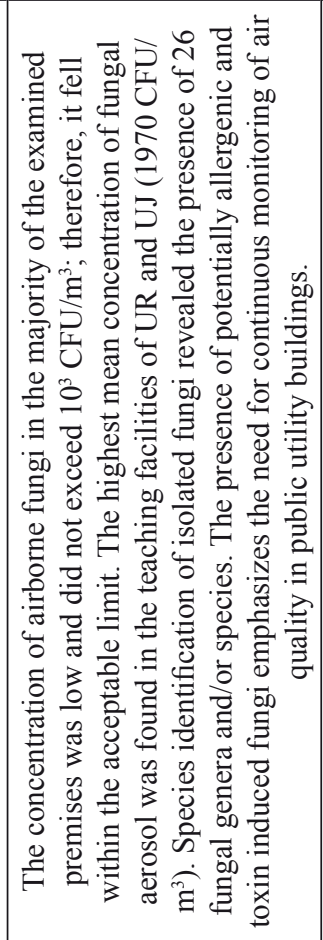 & 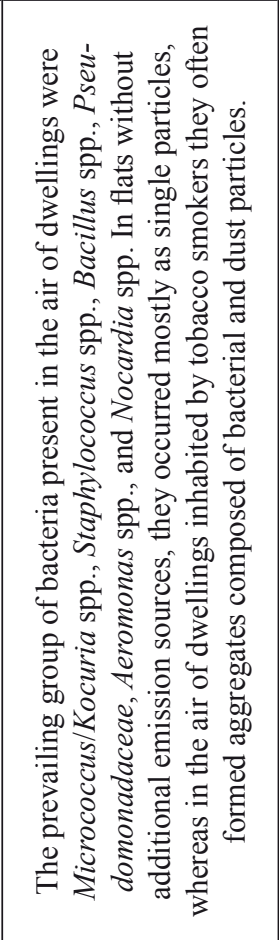 & 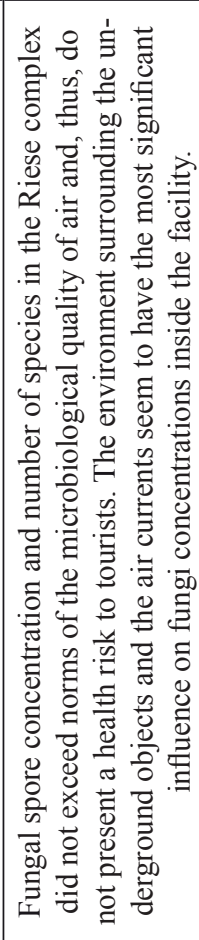 \\
\hline 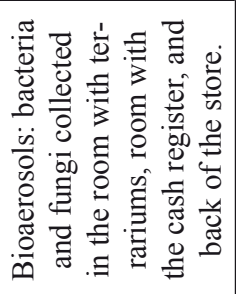 & 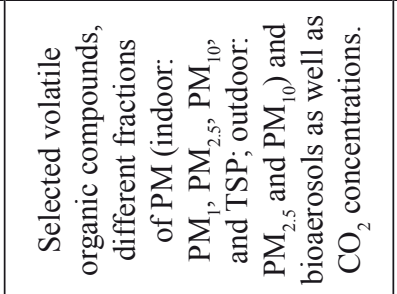 & 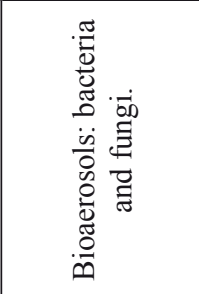 & 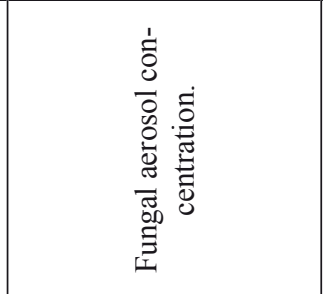 & 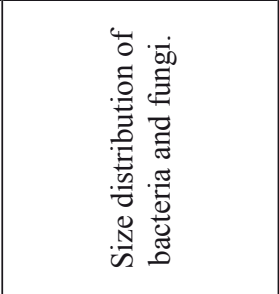 & 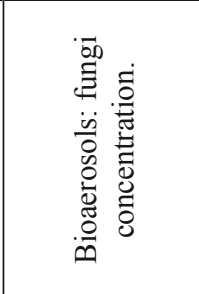 \\
\hline 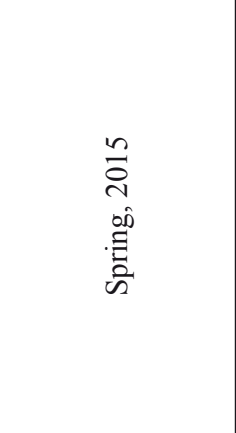 & 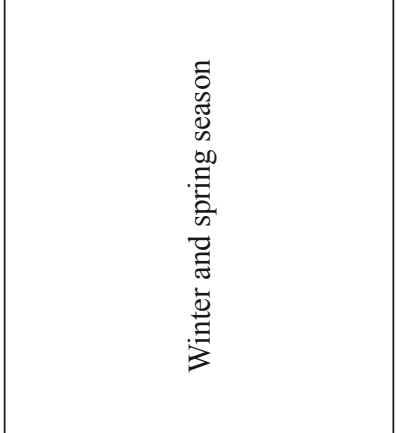 & 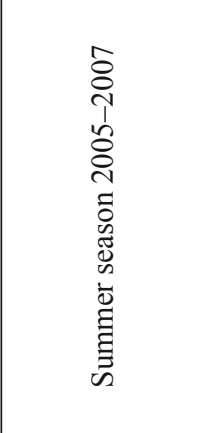 & 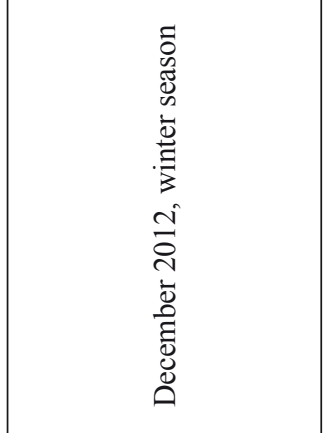 & 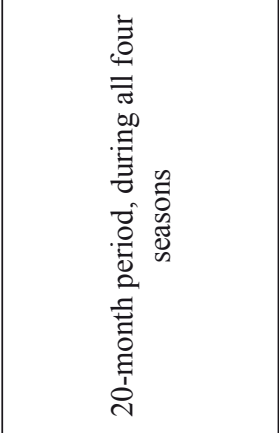 & 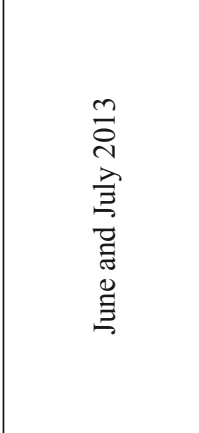 \\
\hline 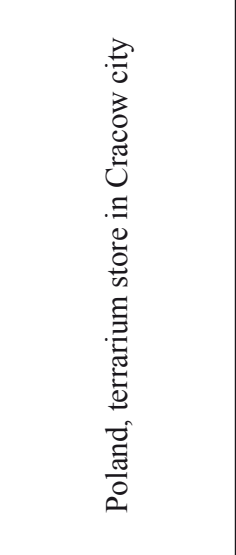 & 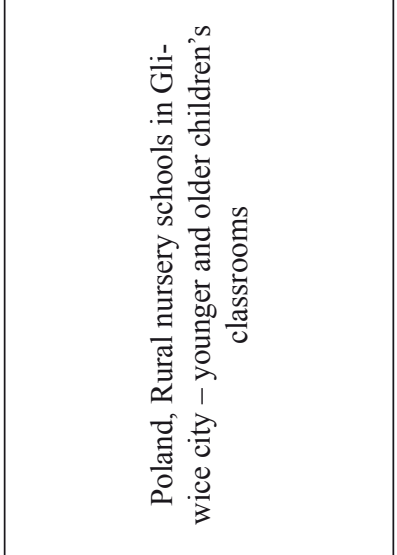 & 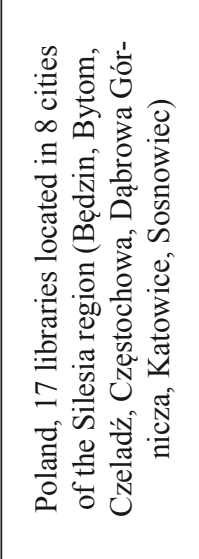 & 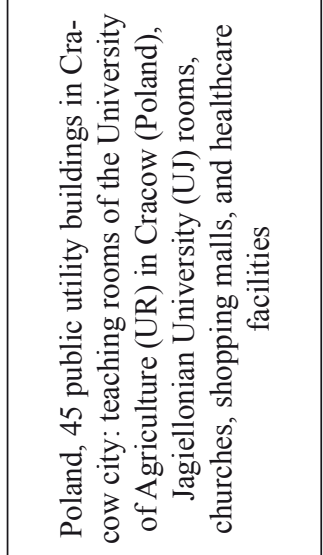 & 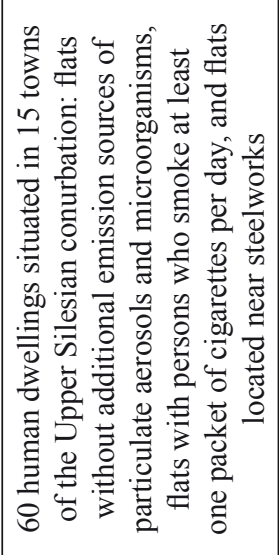 & 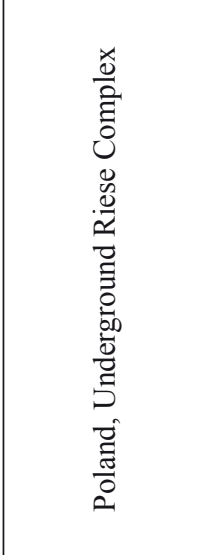 \\
\hline$\underset{\infty}{\widetilde{D}}$ & $\stackrel{\widetilde{\infty}}{\mathscr{\infty}}$ & $\underset{D}{F}$ & $\sqrt[\infty]{\infty}$ & $\begin{array}{l}\square \\
\mathbb{\infty}\end{array}$ & $\underset{\infty}{\sigma}$ \\
\hline$\dot{\sim}$ & $\ddot{a}$ & $\dot{\sim}$ & $\dot{\imath}$ & $\dot{\sim}$ & $\dot{\lambda}$ \\
\hline
\end{tabular}




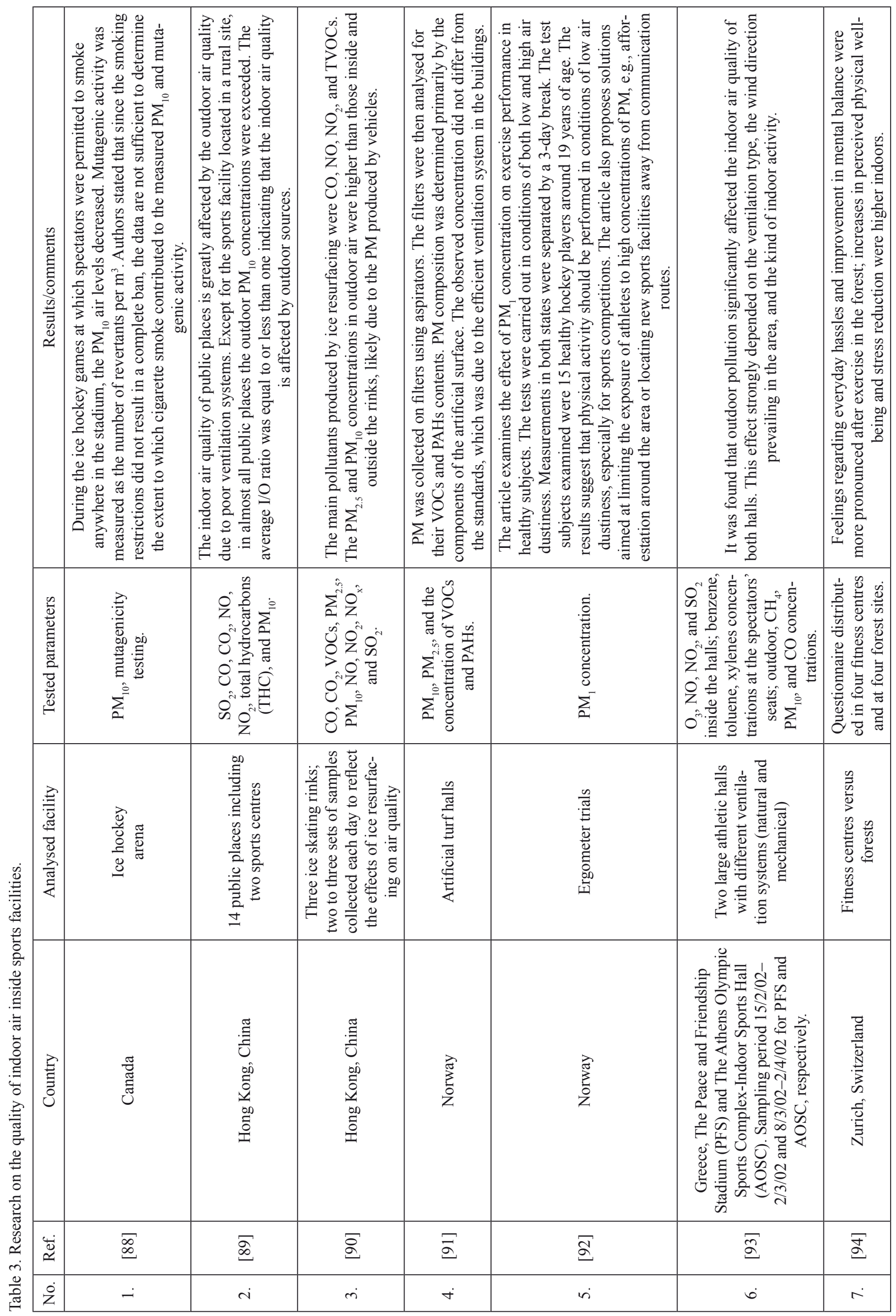




\begin{tabular}{|c|c|c|c|c|c|c|c|}
\hline 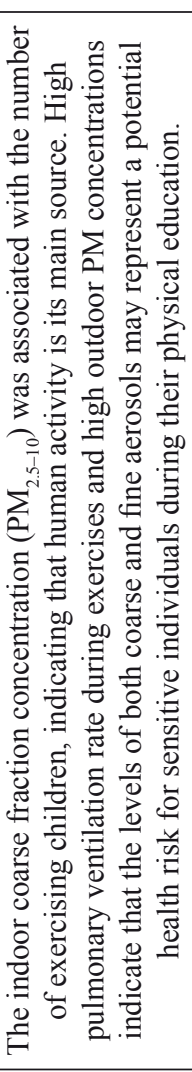 & 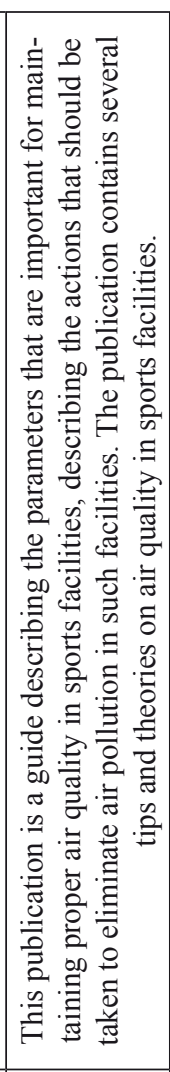 & 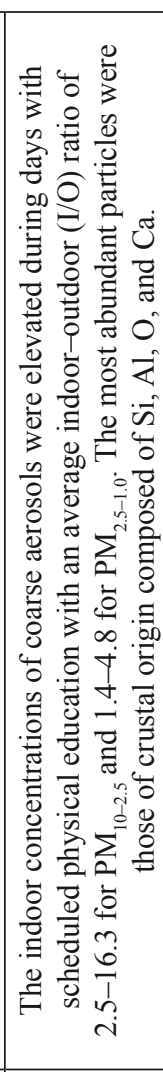 & 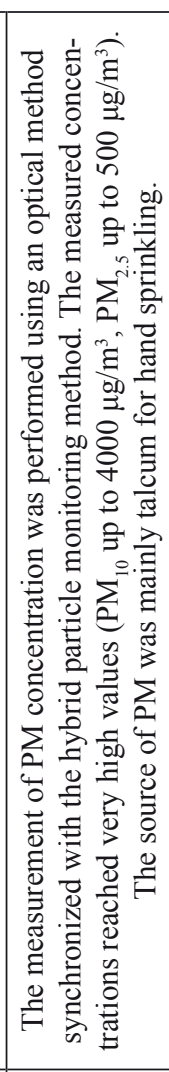 & 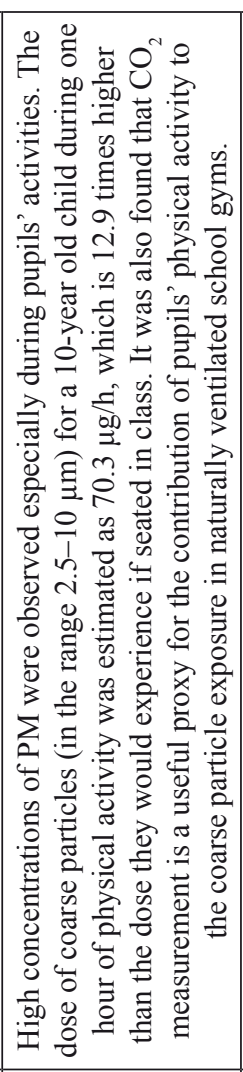 & 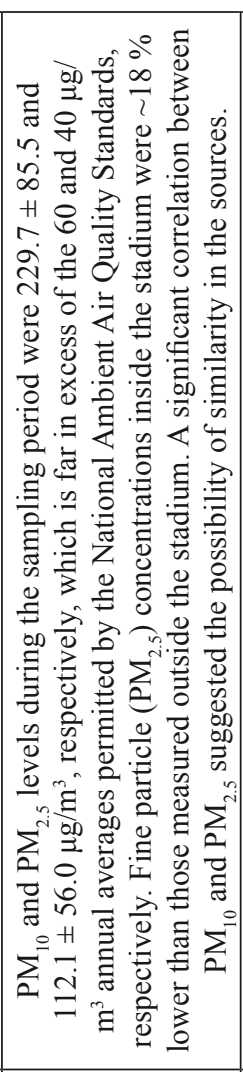 & 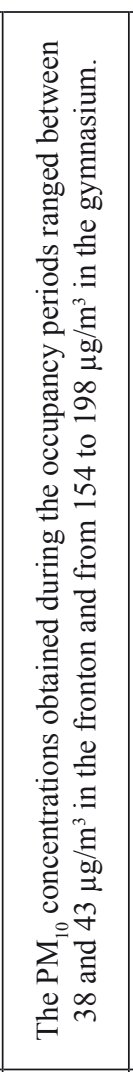 & 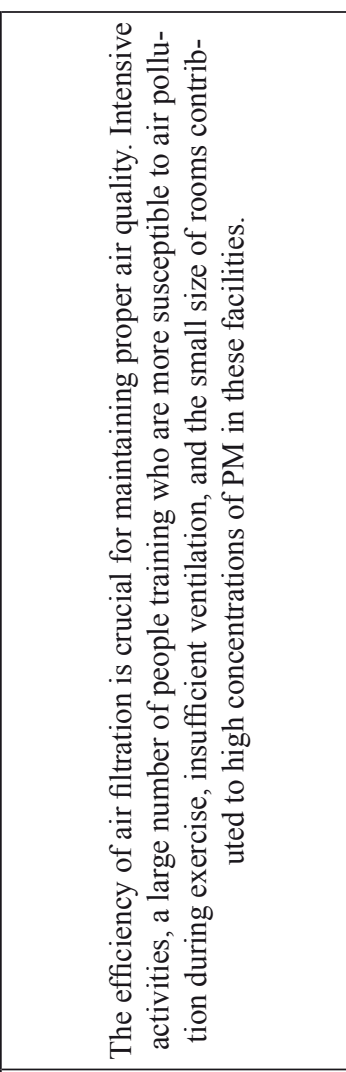 \\
\hline 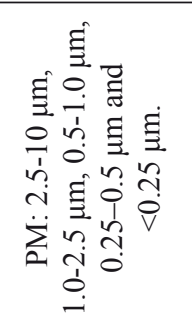 & 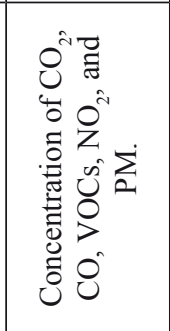 & 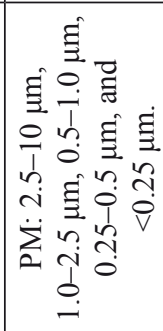 & 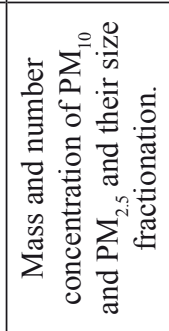 & 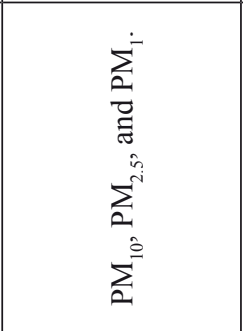 & 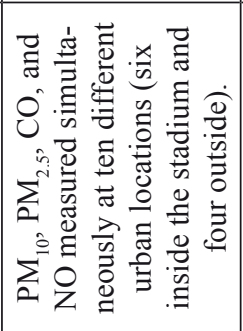 & $\begin{array}{l}\text { च } \\
\text { च } \\
\underbrace{\text { in }} \\
0 \\
0 \\
0 \\
0 \\
0 \\
0^{i}\end{array}$ & 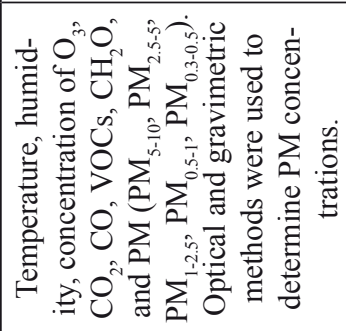 \\
\hline 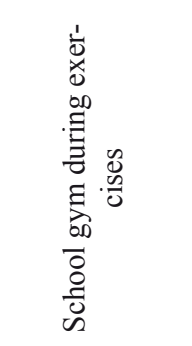 & 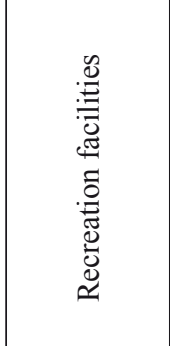 & 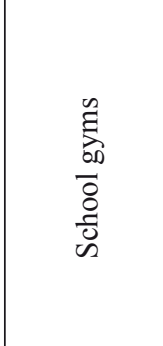 & 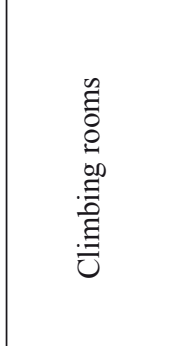 & 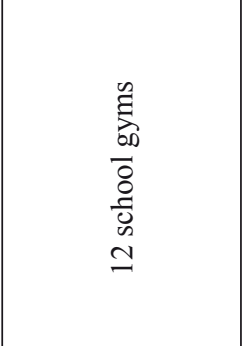 & 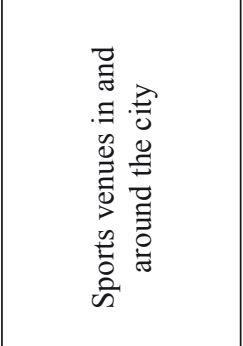 & 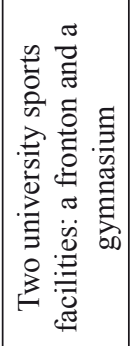 & 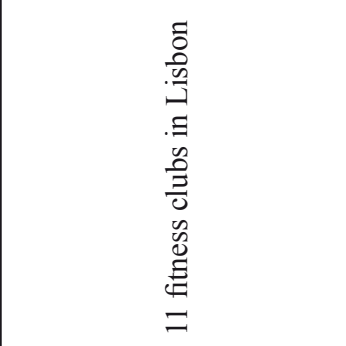 \\
\hline 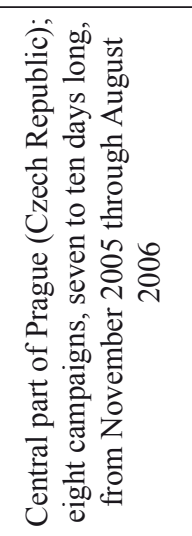 & 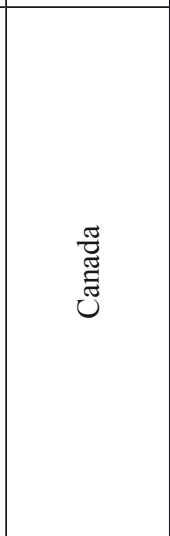 & 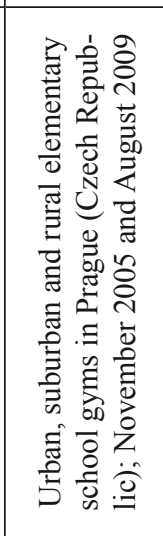 & 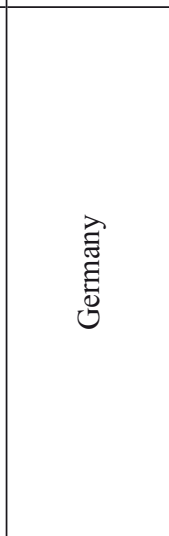 & 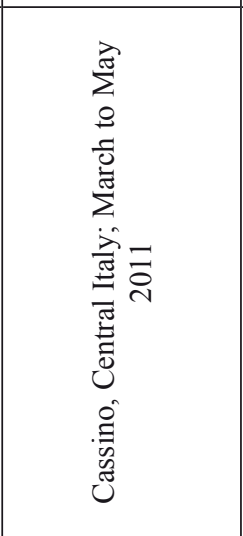 & 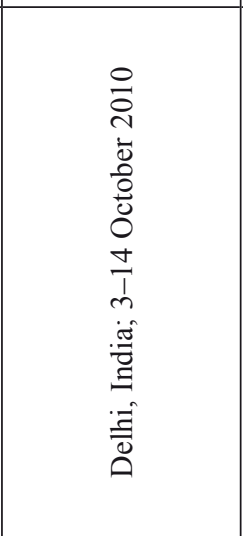 & $\frac{\tilde{n}}{n}$ & 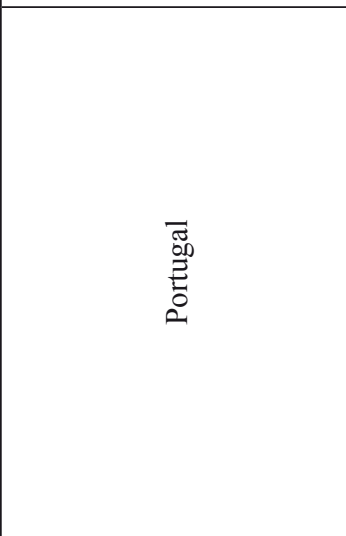 \\
\hline$\check{\Sigma}$ & $\stackrel{\triangleright}{2}$ & $\widehat{\Xi}$ & $\stackrel{\infty}{\varrho}$ & $\bar{\Omega}$ & $\stackrel{\Xi}{\Xi}$ & $\bar{\Xi}$ & $\stackrel{\widetilde{\sigma}}{\varrho}$ \\
\hline$\infty$ & $\sigma^{\circ}$ & $\stackrel{0}{\circ}$ & $\doteq$ & ¿ & $\dot{n}$ & $\dot{I}$ & $\ddot{n}$ \\
\hline
\end{tabular}




\begin{tabular}{|c|c|c|c|c|c|}
\hline 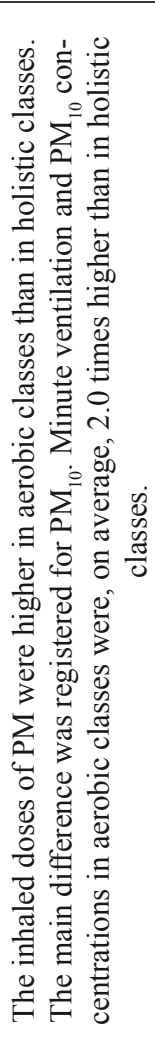 & 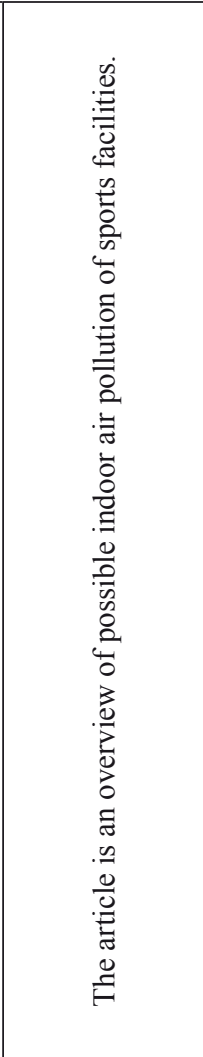 & 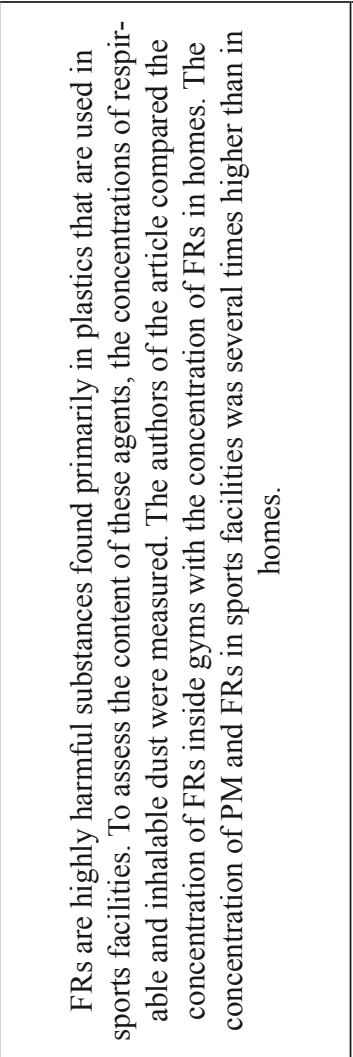 & 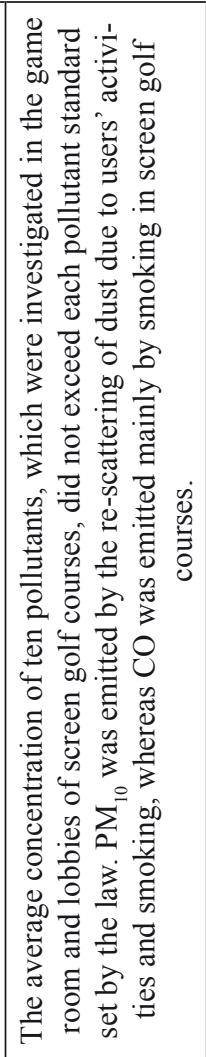 & 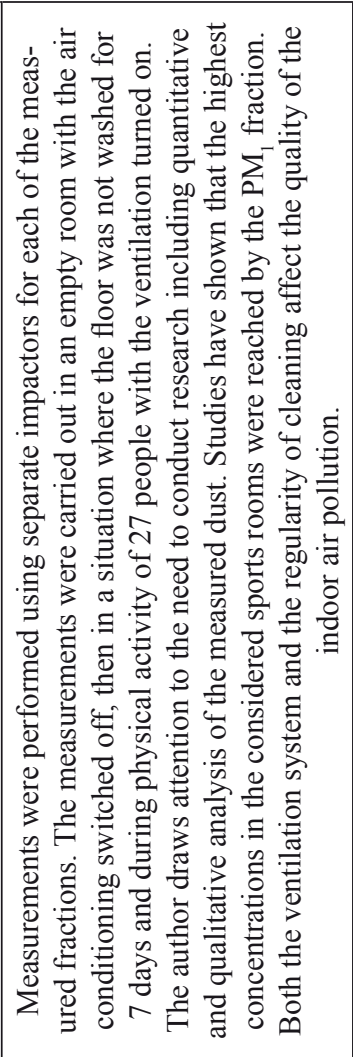 & 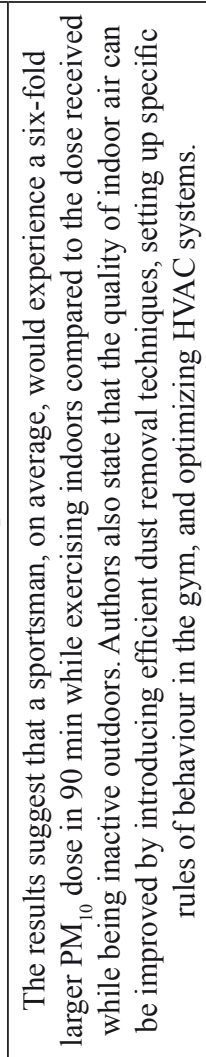 \\
\hline 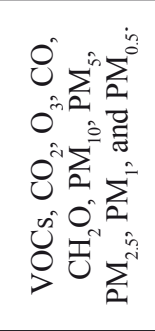 & 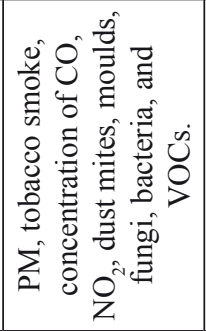 & 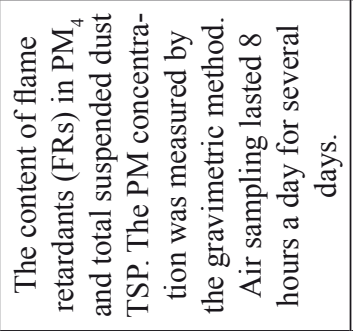 & 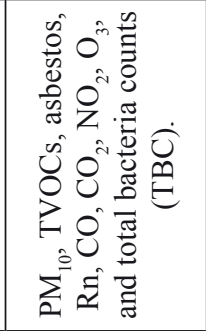 & 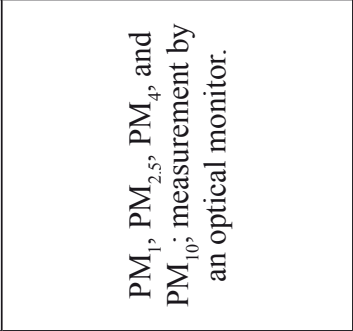 & 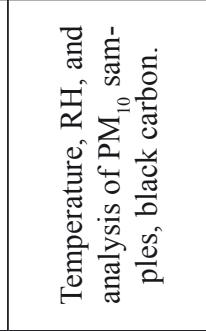 \\
\hline $\begin{array}{l}0 \\
0 \\
0 \\
0 \\
0 \\
0 \\
0 \\
0 \\
0 \\
0 \\
0\end{array}$ & 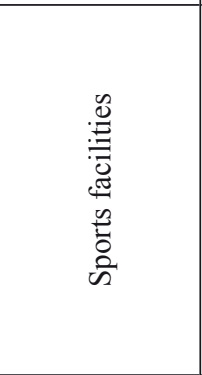 & 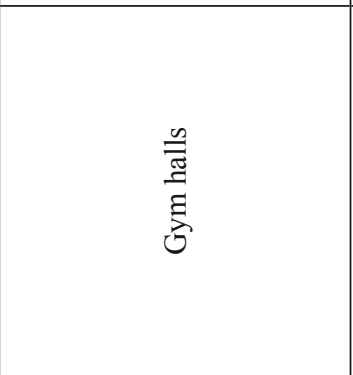 & 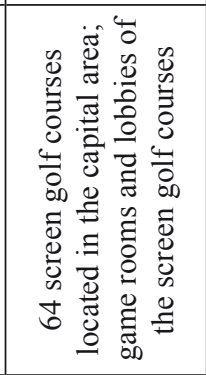 & 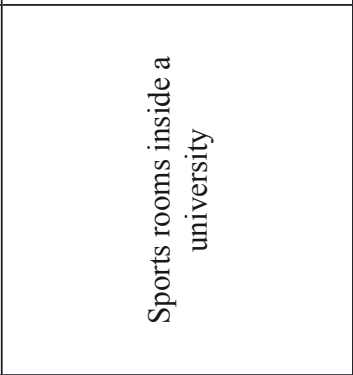 & 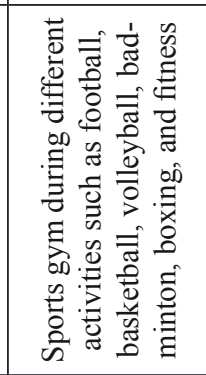 \\
\hline $\begin{array}{l}\overline{\widetilde{g}} \\
\text { 品 } \\
\text { E⿱ }\end{array}$ & 苟 & 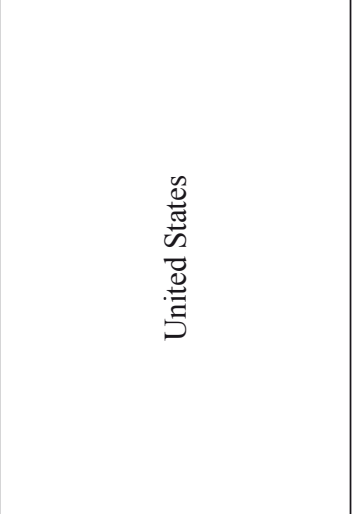 & 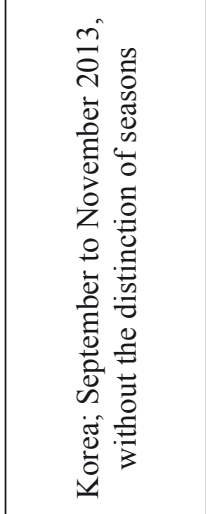 & 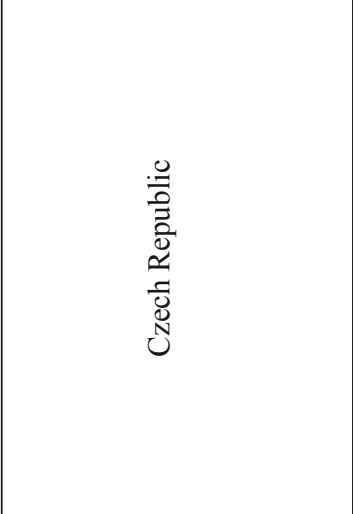 & 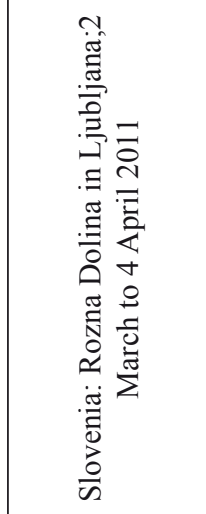 \\
\hline$\underline{\tilde{\varrho}}$ & $\underset{\Xi}{\stackrel{F}{\Xi}}$ & $\stackrel{\check{\varrho}}{\varrho}$ & $\stackrel{\square}{\varrho}$ & $\stackrel{\Xi}{\varrho}$ & $\stackrel{\infty}{\varrho}$ \\
\hline$\stackrel{\bullet}{\circ}$ & $\doteq$ & $\stackrel{\infty}{\sim}$ & $\stackrel{2}{ }$ & $\dot{i}$ & $\dot{\vec{N}}$ \\
\hline
\end{tabular}


about PM harmfulness. Scientific reports concerning the impact of PM on the environment (climate, biosphere, etc.), documenting that the state of indoor air quality in Poland in terms of PM pollution is at least the same or worse as in the atmosphere, as well as progress in measurement methods in recent years, are all driving forces for further research on indoor PM in Poland.

Many works published in recent years relate to the quality of indoor air in non-residential buildings. This is due to the fact that internal sources of PM and emission of PM gas precursors in specific flats and houses are already quite well recognized, and the main difference in air pollution between single houses or even between separate quarters with the same intended use is often a result of their different location. Only in cases where inside residential premises there are some specific sources of PM or PM precursors (i.e., the presence of animals or cigarette smoke, specific cosmetics, or printers) or the room is used in a non-specific way (i.e., very large number of occupants, very large or very low air exchange) may the observed situation in terms of airborne PM pollution as well as its relationship with outdoor (atmospheric) air pollution be specific and worth considering.

Table 1 presents the results of PM measurements in non-residential facilities found in the literature. This is only a small slice of all the works in this field. We selected only those articles that appeared between 2012 and 2017. Our summary also includes all Polish works on air quality in non-residential facilities published in international journals between 2011 and 2018 (Table 2). These articles concern non-residential facilities like offices, universities, or kindergartens, where the mass or number concentration of PM was estimated (Table 2). Some of them also concern the contents of mainly toxic, PM-bound compounds. The largest number of Polish research studies on the quality of indoor air relate to the microbiological cleanliness of the air and hence the concentration of various types of fungi and/or bacteria (Table 2). However, with respect to international research, one can notice some interesting aspects discussed in the research conducted by Polish groups. These include, for example, an interesting location for the measurements, for example in historical buildings, doctor's surgeries, or in an underground health resort.

The research on the properties of PM in various rooms covers a very wide spectrum, including the type of rooms and different PM components as well as quite diverse PM fractions (Table 1). This is due to the fact that from week to week, new research concerning the quality of indoor air appears in the literature. However, despite this, it is still a fraction of all of the results published world-wide on this topic. This short review also shows that only in recent years were some works devoted to the quality of indoor air in sports facilities (Table 3) and the consequences of their pollution on the health of athletes. Facilities of this kind have never been thoroughly examined in terms of the chemical characteristics of the PM pollution, and in Poland the PM concentrations in such a type of facility have not been examined at all.

In the studies of air quality inside sports facilities, authors focus their attention not only on the issues concerning the parameters of internal air but also on aspects of their influence on specific functions of athletes' bodies. In Beijing during the Olympic Games in 2008, high CO concentrations were recorded, which may accelerate the heart rate and decrease the maximal oxygen uptake, potentially penalizing athletic performance, especially in endurance competitions like marathons [109]. The problem of air pollution during the Olympic Games in Beijing was noticed by athletes who during training and leisure time used special facemasks. Several countries located their pre-Olympic training camps outside China, especially in Japan or South Korea. Some athletes even pulled out of the Games due to air pollution [109]. In turn, Bos et. al. investigated the effects of PM exposure during aerobic training on inflammatory biomarkers: the serum brain-derived neurotrophic factor (BDNF), an assumed mediator of exercise-induced cognitive improvements, and cognitive performance [110]. In this study, two groups of volunteers completed aerobic training (lasting 12 weeks, 3 times a week) in two different kinds of area: urban and residential. In the urban environment, the changes in all marker levels after training showed a positive correlation with the personal average exposure to ultrafine particles. Moreover Bos and his team analysed the influence of air pollution on cardiovascular and respiratory function, including exercise capacity [110]. Results were collected from 5604 subjects during the Canada Health Measures Survey. It was found that exposure to higher concentrations of air pollution was associated with higher resting blood pressure and lower ventilatory function, and ozone was associated with reduced exercise capacity.

According to the Small Encyclopedia of Sport [111], a sports facility is a building or place that provides a particular service designed for sports purposes. Generally, we can distinguish two types of sports facilities: indoor facilities - purposely built buildings used for sports like swimming, basketball, badminton, and gymnastics - and outdoor facilities - including pitches (football, rugby, hockey, cricket, etc.), tracks (athletics, horse, dog, and motor racing), purposely built water facilities (rowing, kayaking, sailing), and natural features (like hills and mountains for hiking and climbing and the sea for surfing). The quality of air in outdoor facilities depends on the quality of the atmospheric air and, consequently, on changing weather conditions. A different reasoning applies to closed buildings, where maintaining indoor air quality is done through adequate ventilation, which should provide the facility with an adequate amount of fresh air, guarantee satisfactory thermal comfort and proper 
humidity, adapted to the number of occupants and their physical activity, and should discharge the "used" air outside [112-117]. Other parameters that testify to the quality of indoor air inside sports facilities are the concentration ratio of oxygen to carbon dioxide, the presence of mould and dampness, concentration of VOCs, microbiological contamination, and others [118119].

Two of the most common types of particulate matter inside buildings are thick dust - settled on furniture, floor, room equipment, and different underlying surfaces (its presence is generally associated with mechanical processes, i.e., building demolition, occupant movement, resuspension of retained dust, erosion of equipment, etc.) and fine particles, which can migrate into indoor environments through doors, windows, cracks, leaks, and other unintentional openings in the building envelope [120]. PM is a kind of indoor pollutant that reflects the overall sanitary state of a particular room. Examination of its physicochemical properties allows us to form conclusions about other pollutants, which for example are involved in its formation and transformations. Thanks to the measurements of PM concentration and its chemical and physical characteristics, it is also possible to indicate the general conditions prevailing inside the room (for example by examining the direction and intensity of transformations and reactions of PM gaseous precursors).

An important issue regarding PM particles in sports facilities which shouldn't be ignored is its negative effect on human health. This mostly concerns people who train intensively in conditions of high air dustiness. Fine particles easily get into the lungs and are commonly deposited in the alveoli, ultimately disturbing gas exchange. For athletes training inside dusty facilities, this means that under high concentrations of PM, their performance during activity will be reduced [121]. In addition, during intensive training, most of the inhaled air enters into the body directly through the mouth [122-123], avoiding the clearing mechanisms of the upper respiratory tract - the first line of defence. During normal nasal breathing, the majority of PM particles with dimensions of $2 \mu \mathrm{m}$ and above [108] are practically retained and removed mechanically by nose wiping, blowing, sneezing, etc. As the intensity of exercise increases, the volume, rate, and intensity of breathing also increase. In such cases both coarse and fine particles are transferred into the respiratory tract. The oxygen demand among elite athletes is definitely higher than in the case of any other person. The health benefits of regular exercise and physical activity also include, among others, an increase in the minute tidal volume [124]. During exercise breathing becomes deeper and faster; therefore, athletes are more exposed to air pollution - including PM compared to people who do not engage in any sports activity.

\section{Measurement Problems}

The above tables show that different methods and techniques are used in measuring PM concentrations. The gravimetric method is considered an absolute standard due to the fact that it is a reference method [125-129]. However, there are numerous papers showing the influence of reactions between deposited dust and air, for example, water vapour desorption or absorption of water vapour from air, chemical decomposition of PM-bound gas pollutants (NOx, $\mathrm{SO}_{\mathrm{X}}$, and VOCs), or the influence of the temperature and air humidity on the concentration of dust [130-132]. Moreover, measurements with the standard reference method do not provide the opportunity to track PM concentrations in real time. For these reasons, automatic online monitors with high time resolution are widely used in air pollution measurements, for example the tapered element oscillating microbalance (TEOM - the measurement method consists of the change of frequency of an oscillatory balance) [130, 133-134] or optical meters based on beta attenuation (DustTrak, Eberline - the measurement method is based on the absorption of beta radiation) [102, 135-137]. Nevertheless, due to the vulnerability of optical devices and TEOM to air humidity and seasonal temperature changes which cause overstating of the measurement results, these monitors are not considered as reference methods [138-146]. Due to the wide spectrum of measuring capabilities of automatic monitors, many scientists use them; however, they first conduct equivalence tests between candidate samplers and the gravimetric method. Hauck et al. [134] conducted separate equivalence tests for one year for $\mathrm{PM}_{1}, \mathrm{PM}_{2.5}$, $\mathrm{PM}_{10}$, and TSP fractions in various locations as well as in different measurement seasons (heating and non-heating). Simultaneous with the PM concentrations, measurements of the PM chemical composition, meteorological conditions, and concentrations of gaseous pollutants were analysed. Vardoulakis and Kassomenos, simultaneously with the equivalence tests, also measured other pollutants $\left(\mathrm{NOx}, \mathrm{CO}, \mathrm{O}_{3}\right.$, and $\mathrm{SO}_{2}$ ) and meteorological parameters (wind speed, temperature, relative humidity, precipitation, solar energy, radiation, and atmospheric pressure) to then analyse the correlation between the above parameters and PM concentrations depending on the measurement season [144]. Noteworthy is the long, three-year duration of the measurements (2001-2003).

Gehrig et al. developed a new method combining $\mathrm{PM}_{10}$ data from automatic monitors (beta-attenuation) with a limited number of gravimetric $\mathrm{PM}_{10}$ values which are available for every fourth day from the National Air Pollution Monitoring Network (NABEL) in Switzerland [135]. Measurements were carried out simultaneously in various locations (urban areas, suburbs, highways, street canyons, rural areas). The conditioning and weighing of the filters used at different measurement locations were conducted in 
the same laboratory under the same temperature and humidity conditions. Based on the measurements, the correction coefficients were determined separately for the summer and winter seasons, due to the influence of atmospheric conditions and VOC reactions. Gębicki and Szymańska also presented a lot of information and recommendations regarding the implementation of equivalence tests [137-138]. In their publications, they discussed the reference and non-reference methods for measuring the PM concentration and compared the results of equivalence tests carried out in accordance with the PN-EN 12341 standard and the EU guidebook [129].

In almost all of the above-listed publications, non-reference samplers are recommended. However, the most important condition for their application is equivalence tests and separate determination of the correction factor for the summer and winter seasons. The authors of the studies point out that the distribution of inconsistent results with the gravimetric method creates a double risk. In the case of low results for PM concentrations, the population will be exposed to the deteriorating quality of the environment, affecting its health. On the other hand, inflated results can stimulate the launch of costly correction programmes.

\section{Conclusions}

Generally, in Poland measurements of indoor particulate matter should be conducted much more intensely and on a larger scale than in recent years. Determining the physicochemical properties of PM in closed sports facilities seems to be one of the most important future research problems within the indoor air quality investigation not only in Poland but all over the world. It is also important to determine the sources of PM and its components inside sports facilities and link these parameters with the parameters designated for ambient (atmospheric) PM. The diagnosis of these variables would be beneficial in determining the inhaled doses of PM during exercise, both in terms of singular short-term training but also in a longer time frame (several years) and even during the entire lifetime (athlete exposure scenario), and this would help to estimate a health risk assessment related to exposure to PM and its components. It seems that this problem may be particularly important for closed sports facilities when the existing ventilation system is not adapted to the size of the room and/or the intensity of its use, which may contribute to the accumulation of pollutants inside the facility and thus increase exposure to PM and its components.

Undoubtedly, before undertaking such extensive research, attention should be paid to refining the measurement methodology to the specifics of a given room or, more precisely, suitable for the type and properties of PM in a room.

\section{Acknowledgements}

The authors would like to acknowledge the Ministry of Science and Higher Education for their financial support as part of the statutory works Nos. S/E/422/8/16/17 and S/E-422/32/17.

\section{Conflict of Interest}

The authors declare no conflict of interest.

\section{References}

1. SPURNY K.R. Chemical mixtures in atmospheric aerosols and their correlation to lung diseases and lung cancer occurrence in the general population. Toxicol Lett, 88, 271, 1996.

2. MONN C. Exposure assessment of air pollutants: a review on spatial heterogeneity and indoor/outdoor/personal exposure to suspended particulate matter, nitrogen, dioxide and ozone. Atmos Environ, 35, 1, 2001.

3. WORLD HEALTH ORGANIZATION. Air quality guidelines - global update, Geneva 2005. Available online: http://www.who.int/phe/health_topics/en/ (accessed on 14.03.2018)

4. BROOK R.D. Cardiovascular effects of air pollution. Clinical Science (London), 115, 175, 2008.

5. ANDERSON J.O., THUNDIYIL J.G., STOLBACH A. Clearing the air: a review of the effects of particulate matter air pollution on human health. J Med Toxicol, 8, 166, 2012.

6. EEA's Air quality in Europe - 2016. EEA Report No $5 / 2015$.

7. WATKINSON W.P., CAMPEN M.J., COSTA D.L. Cardiac arrhythmia induction after exposure to residual oil fly ash particles in a rodent model of pulmonary hypertension. Toxicol Sci, 41, 209, 1998.

8. VEDAL S., BRAUER M., WHITE R., PETKAU J. Air pollution and daily mortality in a city with low levels of pollution. Environ Hlth Persp, 111, 45, 2003.

9. BELL M.L., SAMET J.M., DOMINICI F. Time-series studies of particulate matter. Annu Rev Publ Health, 25, 247, 2004.

10. SHI L., ZANOBETTI A., KLOOG I., COULL B.A., KOUTRAKIS P., MELLY S.J., AND SCHWARTZ J.D. Low-concentration $\mathrm{PM}_{2.5}$ and mortality: estimating acute and chronic effects in a population-based study. Environ Hlth Persp, 124, 46, 2016.

11. PENTTINEN P., TIMONEN K. L., TIITTANEN P., MIRME A., RUUSKANEN J., PEKKANEN J. Number concentration and size of particles in urban air: effects on spirometric lung function in adult asthmatic subjects. Environ Hlth Persp, 109, 319, 2001.

12. CARVALHO-OLIVEIRA R., PIRES-NETO R.C., BUSTILLOS J.O., MACCHIONE M., DOLHNIKOFF M., SALDIVA P.H., GARCIA M.L. Chemical composition modulates the adverse effects of particles on the mucociliary epithelium. Clinics, 70, 706, 2015.

13. WILSON J.G., KINGHAM S., PEARCE J., STURMAN A.P. A review of interurban variations in particulate air pollution: implications for epidemiological research. Atmos Environ, 39, 6444, 2005. 
14. PETERS A., WICHMAN H.E., TUCH T., HEINRICH J., HEYDER J. Respiratory effects are associated with the number of ultrafine particles. Am J Resp Crit Care, 155, 1376, 1997.

15. ROGULA-KOZŁOWSKA W., MAJEWSKI G., CZECHOWSKI P.O. The size distribution and origin of elements bound to ambient particles: a case study of a Polish urban area. Environ Monit Assess, 187, 240, 2015.

16. KLEJNOWSKI K., PASTUSZKA J. S., ROGULAKOZŁOWSKA W., TALIK E., KRASA, A. Mass size distribution and chemical composition of the surface layer of summer and winter airborne particles in Zabrze, Poland. B Environ Contam Tox, 88, 255, 2012.

17. KLEJNOWSKI K., KRASA A., ROGULA-KOZLOWSKA W., BŁASZCZAK B. Number size distribution of ambient particles in a typical urban site: the first polish assessment based on long-term (9 Months) measurements. The Scientific World Journal, ID 539568, 2013.

18. ROGULA-KOZŁOWSKA W. Size-segregated urban particulate matter: mass closure, chemical composition, and primary and secondary matter content. Air Qual Atmos Hlth, 9, 533, 2016.

19. BŁASZCZYK E., ROGULA-KOZŁOWSKA W., KLEJNOWSKI K., FULARA I., MIELŻYŃSKA-ŠVACH D. Polycyclic aromatic hydrocarbons bound to outdoor and indoor airborne particles $\left(\mathrm{PM}_{25}\right)$ and their mutagenicity and carcinogenicity in Silesian kindergartens. Air Qual Atmos Hlth, 10, 389, 2016.

20. BEŁDOWSKA M., SANIEWSKA D., FALKOWSKA L., LEWANDOWSKA A. Mercury in particulate matter over polish zone of the Southern Baltic Sea. Atmos Environ, 46, 397, 2012.

21. PYTA H., ROGULA-KOZŁOWSKA W. Determination of mercury in size-segregated ambient particulate matter using CVAAS. Microchem J, 124, 76, 2016.

22. MAJEWSKI G., ROGULA-KOZŁOWSKA W. The elemental composition and origin of fine ambient particles in the largest Polish conurbation: first results from the short-term winter campaign. Theor Appl Clim, 125, 79, 2016.

23. PASTUSZKA J.S., ROGULA-KOZŁOWSKA W., ZAJUSZ-ZUBEK E. Characterization of $\mathrm{PM}_{10}$ and $\mathrm{PM}_{25}$ and associated heavy metals at the crossroads and urban background site in Zabrze, Upper Silesia, Poland, during the smog episodes. Environ Monit Assess, 168, 613, 2010.

24. BŁASZCZAK B., REIZER M., JUDA-REZLER K., KRAJNY E., MATHEWS B., KLEJNOWSKI K. Analysis of national verses long-range transport contribution to organic and inorganic aerosol load in selected location in Poland. Nato-Chal M, XXIV, 65, 2016.

25. BŁASZCZAK B., ROGULA-KOZLOWSKA W., MATHEWS B., JUDA-REZLER K. Chemical compositions of $\mathrm{PM}_{25}$ at two non-urban sites from the polluted region in Europe. Aerosol Air Qual Res, 16, 2333, 2016.

26. JABŁOŃSKA M., JANECZEK J., MAGIERA T. Mineral composition and morphology of magnetic particles in industrial dust. Goldschmidt Conference, 13-18.06.2010, USA.

27. GÓRKA M., RYBICKI M., BERND R., SIMONEIT R.T., MARYNOWSKI L. Determination of multiple organic matter sources in aerosol PM10 from Wrocław, Poland using molecular and stable carbon isotope compositions. Atmos Environ, 89, 739, 2014.
28. SÓWKA I., ZWOŹDZIAK A., TRZEPLA-NABAGLO K., SKRĘTOWICZ M., ZWOŹDZIAK J. PM elemental composition and source apportionment in a residental area of Wrocław, Poland. Environ Prot Eng, 38, 73, 2012.

29. SAMEK L., ZWOŹDZIAK A., SÓWKA I. Chemical characterization and source identification of particulate master PM10 in a rural and urban sites in Poland. Environ Prot Eng, 39, 91, 2013.

30. ROGULA-KOZŁOWSKA W., KOSTRZON M., ROGULA-KOPIEC P., BADYDA A. Particulate Matter in the air of the underground chamber complex of the Wieliczka Salt Mine Health Resort. Adv Exp Med Biol 955, 9, 2016.

31. REIZER M., JUDA-REZLER K. Explaining the high PM concentrations observed in Polish urban areas. Air Qual Atmos Hlth, 9, 517, 2016.

32. KOWALSKA M., KOCOT K. Short-term exposure to ambient fine particulate matter $\left(\mathrm{PM}_{2.5}\right.$ and $\left.\mathrm{PM}_{10}\right)$ and the risk of heart rhythm abnormalities and stroke. Adv Hyg Exp Med, 70, 1017, 2016.

33. SÓWKA I., PACHURKA Ł., PRZEPIÓRKA M., ROGULA-KOZŁOWSKA W., ZWOŹDZIAK A. Evaluation of the short-term impact of particulate matter concentrations on the health of the Wrocław city inhabitants. Annu Set Environ Prot, 18, 603, 2016.

34. WIDZIEWICZ K., LOSKA K. Metal induced inhalation exposure in urban population: a probabilistic approach. Atmos Environ 128, 198, 2016.

35. WIDZIEWICZ K., ROGULA-KOZŁOWSKA W., LOSKA K. Cancer risk from arsenic and chromium species bound to $\mathrm{PM}_{25}$ and $\mathrm{PM}_{1}$ - Polish case study. Atmos Pollut Res, 7, 884,2016

36. ZWOŹDZIAK A., GINI M.I., SAMEK L., ROGULAKOZLOWSKA W., SÓWKA I. Implications of the aerosol size distribution modal structure of trace and major elements on human exposure, inhaled dose and relevance to the $\mathrm{PM}_{2.5}$ and $\mathrm{PM}_{10}$ metrics in a European Pollution Hotspot urban area. J Aerosol Sci 103, 38, 2016.

37. BADYDA A. Zagrożenia środowiskowe ze strony transportu. Nauka, 4, 115, 2010.

38. JANKOWSKA E., WIĘCEK E. Dusts [In Polish: Pyły], CIOP, Warsaw, 2011.

39. WERYSZKO-CHMIELEWSKA E., PIOTROWSKAWERYSZKO K., SULBORSKA A, TIETZE M., MATYSIK-WOŹNIAK A. Biological and particulate pollutants threatening human health [In Polish: Zanieczyszczenia biologiczne i pyłowe w powietrzu atmosferycznym zagrażające zdrowiu człowieka]. Alergoprofil 11, 23, 2015.

40. LI C.S. Relationships of indoor outdoor inhalable and respirable particles in domestic environments. Sci Tot Environ, 151, 205, 1994.

41. LANDSBERGER S., WU D. The impact of heavy metals from environmental tobacco smoke on indoor air quality as determined by Compton suppression neutron activation analysis. Sci Tot Environ, 173, 323, 1995.

42. BAEK S.O., KIM Y.S., PERRY R., Indoor air quality in homes, offices and restaurants in Korean urban areas Indoor/outdoor relationships. Atmos Environ, 31, 529, 1997.

43. MICALLEF A., CALDWELL J., COLLS J.J. The influence of human activity on the vertical distribution of airborne particle concentration in confined environments: Preliminary results. Indoor Air, 8, 131, 1998. 
44. TUNG T.C., CHAO C.Y, BURNETT J. A methodology to investigate the particulate penetration coefficient through building shell. Atmos Environ, 33, 881, 1999.

45. CAMUFFO D., BRIMBLECOMBE P., VAN GRIEKEN R., BUSSE H.J., STURARO G., VALENTINO A., BERNARDI A., BLADES N., SHOOTER D., DE BOCK L., GYSELS K., WIESER M., KIM O. Indoor air quality at the Correr Museum, Venice, Italy. Sci Tot Environ, 236, 135, 1999.

46. BRIMBLECOMBE P., BLADES N., CAMUFFO D., STURARO G., VALENTINO A., GYSELS K., VAN GRIEKEN R., BUSSE H.J., KIM O., ULRYCH U., WIESER $M$. The indoor environment of a modern museum building, the Sainsbury Centre for Visual Arts, Norwich, UK. Indoor Air, 9, 146, 1999.

47. JELEŃSKA M., GÓRKA-KOSTRUBIEC B., KRÓL E. Magnetic properties of dust as indicators of indoor air pollution: Preliminary studies. [In]: Menagement of Indoor Air Qual [Ed. Dudzińska M.], Tylor and Francis Group, 2011.

48. ZWOŹDZIAK A., SÓWKA I., FORTUNA, M., BALIŃSKA-MIŚKIEWICZ, M., WILLAK-JANC, E., \& ZWOŹDZIAK, J. Influence of PM1, PM2.5, PM10 concentrations in indoor school environment on spirometric parameters in schoolchildren. Annu Set Environ Prot, 15, 2022, 2013.

49. POŁEDNIK B. Variations in particle concentrations and indoor air parameters in classrooms in the heating and summer seasons. Arch Environ Prot, 39, 15, 2013.

50. MAINKA A., ZAJUSZ-ZUBEK E. Indoor air quality in urban and rural preschools in Upper Silesia, Poland: particulate matter and carbon dioxide. Int J Environ Res Pub Hlth, 12, 7697, 2015.

51. MAINKA A., ZAJUSZ-ZUBEK E., KACZMAREK K. $\mathrm{PM}_{2.5}$ in Urban and Rural Nursery Schools in Upper Silesia, Poland: Trace Elements Analysis. Int J Environ Res Pub Hlth, 12 (7), 7990, 2015.

52. ROGULA-KOPIEC P., PASTUSZKA J.S., ROGULAKOZŁOWSKA W., MAJEWSKI G. Particulate matter in indoor spaces: known facts and the knowledge gaps, Ann. Warsaw Univ. Life Sci. 43-54, 2015.

53. KOCISZEWSKA K., .ROGULA-KOPIEC P., MAJEWSKI G., ROGULA-KOZŁOWSKA W., MUCHA W., MATHEWS B. Research on carbon occurrence in indoor air of selected universities in Poland. Eng Environ Sci, 26, 108, 2017.

54. AlveS C., CALVO A.I., CASTRO A., FRAILE R., EVTYUGINA M., BATE-EPEY E.F. Indoor air quality in two university sports facilities. Aerosol Air Qual Res, 13, 1723, 2013.

55. HEALTH CANADA. Guidance for fine particulate matter $\left(\mathrm{PM}_{2.5}\right)$ in residential indoor air, 2012. (Available online: https://www.canada.ca/en/health-canada/services/ publications/healthy-living/guidance-fine-particulatematter-pm2-5-residential-indoor-air.html) (accessed on 4.04.2018)

56. WEEKLY K., RIM D., ZHANG L., BAYEN A., M., NAZAROFF W.W., SPANOS C.J. Low-cost coarse airborne particulate matter sensing for indoor occupancy detection, IEEE. Int Conf Automat Sci Eng (CASE), 32, 2013.

57. MOHAMMADYAN M., SHABANKHANI B. Indoor $\mathrm{PM}_{1}, \mathrm{PM}_{25}, \mathrm{PM} 10$ and outdoor $\mathrm{PM}_{25}$ concentrations in primary schools in Sari, Iran. Arch Ind Hyg Tox, 64, 371, 2013.
58. PYKHOVA E. Comparison of indoor air and outdoor air contaminant concentrations, Building Services Engineering, Bachelor's Thesis, 2014.

59. ARAUJO I.P.S., COSTA D.B., MORAES R.J.B. Identification and characterization of Particulate Matter Concentrations at construction jobsites. Sustainability 6, 7666, 2014.

60. FUNK W.E., PLEIL J.D., PEDIT J.A., BOUNDY M.G., YEATTS K.B., NASH D.G., TRENT CH.B., SADIG M., DAVIDSON CH.A., LEITH D. Indoor Air Quality in the United Arab Emirates. J Environ Prot, 5, 709, 2014.

61. MOHDDIN S.A., AMINUDDIN N.M. The exposure assessment of airborne particulates matter $\left(\mathrm{PM}_{10}, \mathrm{PM}_{25}\right)$ towards building occupants: A case study at KL Sentral, Kuala Lumpur, Malaysia. IOP Conf Series, Earth Environ Sci 18, 012008, 2014.

62. ALI M.Y.M., HANAFIAH M.M., LATIF M.T. Composition and distribution of particulate matter $\left(\mathrm{PM}_{10}\right)$ in a mechanically ventilated university building. AIP Conf Proceed, 1784, 060017, 2016.

63. ZWOŹDZIAK A., SÓWKA I., KRUPIŃSKA B., ZWOŹDZIAK J., NYCH A. Infiltration or indoor sources as determinants of the elemental composition of particulate matter inside a school in Wrocław, Poland. Build Environ, 66, 173, 2013.

64. MAJEWSKI G., KOCISZEWSKA K., ROGULAKOZLOWSKA W., PYTA H., ROGULA-KOPIEC P., MUCHA W., PASTUSZKA J.S. Submicron particlebound mercury in university teaching rooms: a summer study from two polish cities. Atmosphere, 7, 117, 2016.

65. POŁEDNIK B., GUZ Ł., SKWARCZYŃSKI M., DUDZIŃSKA M.R. Thermal conditions and perceived air quality in an air-conditioned auditorium in Lublin, Poland, AIP Conf Proceed, 1752, 040024, 2016.

66. POŁEDNIK B., POŁEDNIK A. Particle concentrations in a church during different weather conditions - a case study. J Ecol Eng 17, 173, 2016.

67. LENART M., OLSZOWSKI T. Indoor-outdoor relations for $\mathrm{PM}_{10}$ mass concentration based on university building. Ecol Chem Eng, 23, 127, 2016.

68. BŁASZCZYK E., ROGULA-KOZŁOWSKA W., KLEJNOWSKI K., KUBIESA P., FULARA I., MIELŻYŃSKA-ŠVACH D. Indoor air quality in urban and rural kindergartens: short-term studies in Silesia, Poland. Air Qual Atmos Hlth, 10, 1207, 2017.

69. ROGULA-KOPIEC P., PASTUSZKA J.S., ROGULAKOZŁOWSKA W., MUCHA W. Organic and elemental carbon bound to particulate matter in the air of printing office and beauty salon. E3S Web of Conferences 22, 00147, 2017.

70. ROGULA-KOPIEC P., PASTUSZKA J.S., MATHEWS B., WIDZIEWICZ K. Factors determining the concentration and chemical composition of particulate matter in the air of selected service facilities. E3S Web of Conferences $\mathbf{2 8}$, 01032, 2018.

71. KUSKOWSKA K., ROGULA-KOZŁOWSKA W., ROGULA-KOPIEC P. Particulate matter and polycyclic aromatic hydrocarbons in a selected athletic hall: ambient concentrations, origin and effects on human health. E3S Web of Conferences 28, 01020, 2018.

72. KARWOWSKA E. Microbiological air contamination in some educational settings. Pol J Environ Stud, 12, 181, 2003.

73. STRYJAKOWSKA-SEKULSKA M., PIOTRASZEWSKAPAJĄK A., SZYSZKA A., NOWICKI M., FILIPIAK M. 
Microbiological quality of indoor air in university rooms. Pol J Environ Stud, 16, 623, 2007.

74. WÓJCIK A., CHORĄŻY L., MITUNIEWICZ T., WITKOWSKA D., IWAŃCZUK-CZERNIK K., SOWIŃSKA J. Microbial air contamination in poultry houses in the summer and winter, Pol J Environ Stud, 19, 1045, 2010.

75. GOŁOFIT-SZYMCZAK M., GÓRNY R. Bioaerosols in residential homes (In Polish: Bioaerozole w budynkach biurowych). Kosmos, 66, 491, 2017.

76. KALWASIŃSKA A., BURKOWSKA A., WILK I. Microbial air contamination in indoor environment of a university library. Ann Agr Env Med, 19, 25, 2012.

77. DUMAŁA S.M., DUDZIŃSKA M.R. Microbiological indoor air quality in polish schools. Annu Set Environ Prot, 15, 231, 2013.

78. POŁEDNIK B. Aerosol and bioaerosol particles in a dental office. Environ Res, 134, 405, 2014.

79. KARBOWSKA-BERENT J., GORNY R.L., STRZELCZYK A.B., WLAZŁO A. Airborne and dust borne microorganisms in selected Polish libraries and archives. Build Environ 46, 1872, 2011.

80. PASTUSZKA J.S., KYAW THA PAW U., LIS D.O., WLAZŁO A., ULFIG K. Bacterial and fungal aerosol in indoor environment in Upper Silesia, Poland. Atmos Environ 34, 3933, 2000.

81. EJDYS E., MICHALAK J., SZEWCZYK K.M. Yeastlike fungi isolated from indoor air in school buildings and the surrounding outdoor air. Acta Mycologica, 44, 97, 2009.

82. BULSKI K., KORTA-PEPŁOWSKA M. Microbiological air quality in the terraristic store. Water-Environ-Rural Areas, 17, 27, 2017.

83. MAINKA A., ZAJUSZ-ZUBEK E., KOZIELSKA B., BRACGOSZEWSKA E. Investigation of air pollutants in rural nursery school - a case study. E3S Web of Conferences, 28, 01022, 2018.

84. WLAZŁO A., GÓRNY R.L., ZŁOTKOWSKA R., ŁAWNICZEK A., ŁUDZEŃ-IZBIŃSKA B., HARKAWY A.S., ANCZYK E. Workers exposure to selected biological agents in libraries of Upper Silesia, Occup Med, 59, 159, 2008

85. LENART-BOROŃ A., WOLNY-KOŁADKA K. Fungal aerosol in public utility buildings in the city of Kraków. Environ Med, 18, 18, 2015.

86. GÓRNY R.L., DUTKIEWICZ J., KRYSIŃSKATRACZYK E. Size distribution of bacterial and fungal bioaerosols in indoor air. Ann Agr Env Med, 6, 105, 1999

87. OGÓREK R., LEJMAN A. Speleomicological research in selected objects of a underground Riese Complex (Sowie Mountains, Lower Silesia, Poland). Postępy Mikrobiologii, 54, 344, 2015.

88. GEORGHIOU P.E., BLAGDEN P.A., SNOW D.A., WINSOR L., WILLIAMS D.T. Air levels and mutagenicity of $\mathrm{PM}_{10}$ in an indoor ice arena. J Air Pollut. 12, 1583, 1989.

89. LEE S.C., CHAN L.Y., CHIU M.Y. Indoor and outdoor air quality investigation at 14 public places in Hong Kong. Environ Int, 25, 443, 1999.

90. GUO H., LEE S., CHAN L. Indoor air quality in ice skating rinks in Hong Kong. Environ Res 3, 327, 2004.

91. DYE C., BJERKE A., SCHMIDBAUER N., MANØ S. Measurement of air pollution in artificial turf halls. Norwegian Institute for Air Research (NILU). Report NILU OR 03/2006.
92. RUNDELL K.W., CAVISTON R. Ultrafine and fine particulate matter inhalation decreases exercise performance in healthy subjects. J Strength Cond Res, 22, $2,2008$.

93. STATHOPOULOU O., ASSIMAKOPOULOS V., FLOCAS H., HELMIS C. An experimental study of air quality inside large athletic halls. Build Environ, 5, 834, 2008.

94. HUG S.M., HANSMANN R., MONN C., KRÜTLI P., SEELAND K., Restorative effects of physical activity in forests and indoor settings. Int J Fit 4, 25, 2008

95. BRANIŠ M., ŠAFRÁNEK J., HYTYCHOVÁ A. Exposure of children to airborne particulate matter of different size fractions during indoor physical education at school. Build Environ, 44, 1246, 2009.

96. ONTARIO RECREATION FACILITIES ASSOCIATION INC., Guidelines For Indoor Air Quality Arenas, 2011. Available online: http://healthunit.org/wp-content/ uploads/Guidelines_For_Indoor_Air_Quality_In_ Arenas.pdf/ (accessed: 11.03.2018).

97. BRANIŠ, M. AND ŠAFRÁNEK, J. Characterization of Coarse Particulate Matter in School Gyms. Environ Res, 111, 485, 2011.

98. WEINBRUCH S., DIRSCH T., KANDLER K., EBERT M., HEIMBURGER G., HOHENWARTER F. Measurement of air pollution in indoor artificial turf halls. State Programme for Pollution Monitoring, 2012. Available online: http://www.greenkeeper.nl/ upload/digitaal_archief/1253_0111-TA2148-2006_Measurement_air_of_indoor_turf_pich.pdf/_ (accessed: 14.03.2018).

99. BUONANNO G., FUOCO F., MARINI S., STABILE L. Particle resuspension in school gyms during physical activities. Aerosol Air Qual Res, 5, 803, 2012.

100. BISHT, D., TIWARI, S., SRIVASTAVA, A., SRIVASTAVA, M.K. Assessment of air quality during 19th common wealth games at Delhi, India. National Hazards, 2, 141, 2013.

101. ALVES C.A., CALVO A.I., CASTRO A., FRAILE R., EVTYUGINA M., BATE-EPEY E.F. Comfort parameters and Particulate Matter $\left(\mathrm{PM}_{10}\right.$ and $\left.\mathrm{PM}_{25}\right)$ in school classrooms and outdoor air. Aerosol Air Qual Res, 13, 1521, 2013.

102. RAMOS C.A., WOLTERBEEK H.T., ALMEIDA S.M. Exposure to indoor air pollutants during physical activity in fitness centers. Build Environ 82, 349, 2014

103. RAMOS C.A., REIS J.F., ALMEIDA T., ALVES F., WOLTERBEEK H.T., ALMEIDA S.M. Estimating the inhaled dose of pollutants during indoor physical activity. Sci Tot Environ, 527-528, 111, 2015.

104. HAJIAN M., MOHAGHEGH S. Indoor air pollution in exercise centers. International. J Med Tox Forensic Med, 5, 22, 2015.

105. GUARDIA M.J., HALE R.C. Halogenated flameretardant concentrations in settled dust, respirable and inhalable particulates and polyurethane foam at gymnastic training facilities and residences. Environ Int, 79, 106, 2015.

106. GOUNG S.J.N., YANG J., KIM Y.S., LEE C.M. A pilot study of indoor air quality in screen golf courses. Environ Sci Pollut Res, 9, 7176, 2015.

107. KIC P. Dust pollution in the sport facilities. Agronomy Res, 14, 75, 2016.

108. ZITNIK, M., BUCAR, K., HITI, B., BARBA Z., RUPNIK Z., ZALOZNIK A., ZITNIK E. RODRIGUEZ 
L., MIHEVC I., ZIBERT J. Exercise-induced effects on a gym atmosphere. Indoor Air 26, 468, 2016.

109. LIPPI G., GUIDI G.C., MAFFULLI N. Air pollution and sports performance in Beijing. Int J Sports Med, 29, 696, 2008.

110. BOS I., DE BOEVER P., VANPARIJS J., PATTYN N., PANIS L.I., MEEUSEN R. Subclinical effects of aerobic training in urban environment. Medecine \& Science in Sports \& Exercise, 45, 439, 2013.

111. GŁUSZEK Z., SKRZYPEK E. Little encyclopedy of sport (In Polish: Mała Encyklopedia Sportu) 1, Sport i Turystyka, 1987.

112. CAKMAK S., DALES R., LEECH J., LIU L. The influence of air pollution on cardiovascular and pulmonary function and exercise capacity: Canadian Health Measures Survey (CHMS). Environ Res, 111, 1309, 2011.

113. FANGER P.O., POPIOŁEK Z., WARGOCKI P. Indoor Environment. The influence on health, comfort and work efficiency. (In Polish: Środowisko wewnętrzne. Wpływ na zdrowie komfort i wydajność pracy). Silesian University of Technology, Gliwice, 2003.

114. HENDIGER J., ZIĘTEK P., CHLUDZIŃSKA M. Ventilation and climatization (In Polish: Wentylacja i klimatyzacja). Materiały pomocnicze do projektowania. Venture Industries, Warsaw, 2009.

115. RECKNAGEL H., SPRENGER E., SCHRAMEK E.R. The compendium of knowledge. Heating, airconditioning, hot water, refrigeration. (In Polish: Kompendium wiedzy. Ogrzewnictwo, klimatyzacja, ciepła woda, chłodnictwo). Omni Scala, Wrocław, 2008.

116. POŚNIAK M., MAKHNIASHVILI I., KOZIEŁ E., JANKOWSKA E. Chemical pollution in office rooms - exposure assessment, (In Polish: Zanieczyszczenia chemiczne w pomieszczeniach pracy biurowej - ocena narażenia). Work Safety 6, 21, 2004.

117. ZABIEGAŁA B., PARTYKA M., NAMIEŚNIK J. Indoor air quality - analysis and monitoring. (In Polish: Jakość powietrza wewnętrznego - analiza i monitoring). Nowe horyzonty i wyzwania $\mathrm{w}$ analityce i monitoringu środowiskowym. CEEAM, Gdańsk, 540, 2003.

118. FERDYN-GRYGIEREK J. Air quality and ventilation in educational buildings. (In Polish: Jakość powietrza i wentylacja w budynkach edukacyjnych). Scientific papers of the Silesian University of Technology (Architecture), 47, 57, 2008.

119. NATHANSON T. Indoor air quality in office buildings: a technical guide a report of the Federal-Provincial Advisory Committee on Environmental and Occupational Health, Canada, 1995.

120. MORAWSKA L., SALTHAMMER, T. Indoor environment: airborne particles and settled dust, John Wiley \& Sons, ISBN:9783527305254, 2006.

121. MENTEŞE S., ARISOY M., RAD A.Y., GÜLLÜ G. Bacteria and fungi levels in various indoor and outdoor environments in Ankara, Turkey. Clean 37, 487, 2009.

122. NIINIMAA V., COLE P., MINTZ S. The switching point from nasal to oronasal breathing. Respir Physiol, 42, 61, 1980

123. CalEPA- California Environmental Protion Agency. Air Resources Board. Short-term health effects associated with exposure to air pollution during exercise. California 2, 2008.

124. SRIKANTH P., SUDHARSANAM S., STEINBERG R. Bio-aerosols in indoor environment: composition, health effects and analysis. Indian J Med Microbiol, 26, 302, 2008.

125. Federal Register. Revised requirements for designation of reference and equivalent methods for PM2.5 and ambient air quality surveillance for particulate matter, Final Rule: 40 CFR Parts 53 and 58, Federal Register 62, 38764, 1997.

126. Federal Register. Federal Register, 55, 43406, 1990.

127. EC. Council directive 1999/30/EC relating to limit values for sulphur dioxide, nitrogen oxide and oxides of nitrogen, particulate matter and lead in ambient air. Official Journal of the European Communities, L163, 41, 1999.

128. NOBLE C.A., VANDERPOOL R.W., PETERS T.M., MCELROY F.F., GEMMILL D.B., WIENER R.W. Federal reference and equivalent methods for measuring fine particulate matter. Aerosol Sci Technol, 34, 457, 2001.

129. CEN. Air Quality - Determination of the PM10 fraction of suspended particulate matter - Reference method and field test procedure to demonstrate reference equivalence of measurement methods. EN 12341, 1998.

130. Regulation of Minister of the Environment of $17^{\text {th }}$ December 2008 (Journal of Laws of the Republic of Poland 2009 No. 5, Item. 31).

131. ALLEN G., SIOUTAS C., KOUTRAKIS P., REISS R., LURMAN F.W., ROBERTS P.T. Evaluation of the TEOM method for measurement of ambient particulate mass in urban areas. J Air Waste Manag Assoc., 47, 682, 1997.

132. EISNER A.D., WIENER R. W. Discussion and evaluation of the volatility test for equivalency of other methods to the federal reference method for fine particulate matter. Aerosol Sci Technol, 36, 433, 2002.

133. PANG Y., EATOUGH N.L., EATOUGH D.J. PM semivolatile organic material at riverside, California: implications for the PM2.5 federal reference method sample. Aerosol Sci Technol, 36, 277, 2002.

134. HAUCK H., BERNER A., GOMISCEK B., STOPPER S., PUXBAUM, H., KUNDI M., PREINING O. On the equivalence of gravimetric PM data with TEOM and beta-attenuation measurements. J Aerosol Sci, 35, 1135, 2004.

135. GEHRIG R., HUEGLIN CH., SCHWARZENBACH B., SEITZ T., BUCHMANN B. A new method to link PM10 concentrations from automatic monitors to the manual gravimetric reference method according to EN12341. Atmos Environ, 39, 2213, 2005.

136. FROMME H., TWARDELLA D., DIETRICH S., HEITMANN D., SCHIERL R., LIEBL B., RUDEN H. Particulate matter in the indoor air of classrooms exploratory results from Munich and surrounding area. Atmos Environ, 41, 854, 2007.

137. GĘBICKI J., SZYMAŃSKA K. Comparison of Tests for Equivalence of Methods for Measuring $\mathrm{PM}_{10}$ Dust in Ambient Air. Pol J Environ Stud, 20, 1465, 2011.

138. GĘBICKI J., SZYMAŃSKA K. Comparative field test for measurement of PM10 dust in atmospheric air using gravimetric (reference) method and b-absorption method (Eberline FH 62-1). Atmos Environ, 54, 18, 2012.

139. CHUNG A., CHANG D., KLEEMAN M., PERRY K., CAHILL T., DUTCHER D., STROUD K. Comparison of real-time instruments used to monitor airborne particulate matter. J Air Waste Manag Assoc, 51, 109, 2001.

140. KIM J., MAGARI S., HERRICK R., SMITH T., CHRISTIANI D. Comparison of fine particle 
measurements from a direct-reading instrument and a gravimetric sampling method. J Occup Environ Hyg, 1, 707, 2004

141. KINGHAM S., DURAND M., ABERKANE T., HARRISON J., GAINES WILSON J., EPTON M. Winter comparison of TEOM. MiniVol and DustTrak $\mathrm{PM}_{10}$ monitors in a wood smoke environment. Atmos Environ, 40, 338, 2006

142. MOOSMÜLLER H., ARNOTT W., ROGERS C., BOWEN J., GILLIES J., PIERSON W., NORBECK J. Time resolved characterization of diesel particulate emissions. 1. Instruments for particle mass measurements. Environ Sci Technol, 35, 781, 2001.

143. YANOSKY J., WILLIAMS P., MACINTOSH D. A comparison of two direct-reading aerosol monitors with the federal reference method for $\mathrm{PM}_{2,5}$ in indoor air. Atmos Environ, 36, 107, 2002.

144. ZHU Y., SMITH T., DAVIS M., LEVY J., HERRICK R., JIANG H. Comparing gravimetric and real-time sampling of PM2.5 concentrations inside truck cabins. J Occup Environ Hyg, 8, 662, 2011.

145. VARDOULAKIS S., KASSOMENOS P. Sources and factors affecting PM10 levels in two European cities: Implications for local air quality management. Atmos Environ, 42, 3949, 2008.

146. Demonstration of Equivalence of Ambient Air Monitoring Methods. EC Working Group on Guidance for the Demonstration of Equivalence, 2005. 